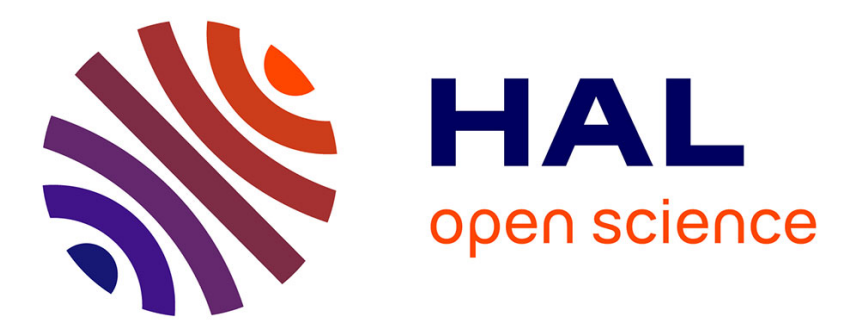

\title{
Logarithmic stability in determination of a 3D viscoelastic coefficient and a numerical example
}

\author{
Maya de Buhan, Axel Osses
}

\section{To cite this version:}

Maya de Buhan, Axel Osses. Logarithmic stability in determination of a 3D viscoelastic coefficient and a numerical example. Inverse Problems, 2010, 26, pp.95006. 10.1088/0266-5611/26/9/095006 . hal-01116979

\author{
HAL Id: hal-01116979 \\ https://hal.science/hal-01116979
}

Submitted on 27 Feb 2015

HAL is a multi-disciplinary open access archive for the deposit and dissemination of scientific research documents, whether they are published or not. The documents may come from teaching and research institutions in France or abroad, or from public or private research centers.
L'archive ouverte pluridisciplinaire HAL, est destinée au dépôt et à la diffusion de documents scientifiques de niveau recherche, publiés ou non, émanant des établissements d'enseignement et de recherche français ou étrangers, des laboratoires publics ou privés. 


\title{
Logarithmic stability in determination of a $3 \mathrm{D}$ viscoelastic coefficient and a numerical example
}

\author{
Maya de Buhan ${ }^{1,2}$ and Axel Osses ${ }^{2}$ \\ ${ }^{1}$ UPMC Univ Paris 06, UMR 7598, Laboratoire J.L. Lions, F-75005 Paris, \\ France \\ 2 Departamento de Ingeniería Matemática and Centro de Modelamiento \\ Matemático (UMI 2807 CNRS), FCFM Universidad de Chile, Casilla 170/3 - \\ Correo 3, Santiago, Chile \\ E-mail: debuhan@ann.jussieu.fr, axosses@dim.uchile.cl
}

\begin{abstract}
We prove a Carleman estimate and a logarithmic stability estimate for an inverse problem in three dimensional viscoelasticity. More precisely, we obtain logarithmic stability for the inverse problem of recovering the spatial part of a viscoelastic coefficient of the form $p(x) h(t)$ from a unique measurement on an arbitrary part of the boundary. The main assumptions are: $h^{\prime}(0)=0, h(0) \neq 0$, $p$ is known in a neighborhood of the boundary and regularity and sensitivity of the reference trajectory. We propose a method to solve the problem numerically and illustrate the theoretical result by a numerical example.
\end{abstract}

PACS numbers: $00.00,20.00,42.10$

Submitted to: Inverse Problems

\section{Introduction and main results}

This article is concerned with the inverse problem of determining an unknown coefficient in the $3 \mathrm{D}$ viscoelasticity system. We set $\Omega$ an open bounded domain of $\mathbb{R}^{3}$ with a sufficiently smooth boundary $\partial \Omega$. The viscoelasticity system, endowed with initial and boundary conditions, is then the following:

$$
\begin{cases}\mathcal{P} u(x, t)=f(x, t), & \forall(x, t) \in \Omega \times(0,+\infty), \\ u(x, 0)=\bar{u}_{0}(x), & \forall x \in \Omega, \\ \partial_{t} u(x, 0)=\bar{u}_{1}(x), & \forall x \in \Omega, \\ u(x, t)=0, & \forall(x, t) \in \partial \Omega \times(0,+\infty),\end{cases}
$$

where $\mathcal{P}$ is the integro-differential hyperbolic operator defined as

$$
\begin{aligned}
& \mathcal{P} u(x, t)=\partial_{t}^{2} u(x, t)-\nabla \cdot\left(\mu(x)\left(\nabla u(x, t)+\nabla u(x, t)^{T}\right)+\lambda(x)(\nabla \cdot u)(x, t) I\right) \\
& \quad+\int_{0}^{t} \nabla \cdot\left(\tilde{\mu}(x, s)\left(\nabla u(x, t-s)+\nabla u(x, t-s)^{T}\right)+\tilde{\lambda}(x, s)(\nabla \cdot u)(x, t-s) I\right) d s .
\end{aligned}
$$

This system models the dynamics of a 3D viscoelastic material subjected to a load $f, u$ being the displacement vector, $\bar{u}_{0}$ and $\bar{u}_{1}$ the initial displacement and velocity, 
$(\lambda, \mu)$ the Lamé coefficients and $(\tilde{\lambda}, \tilde{\mu})$ the viscosity coefficients, respectively. The well-posedness nature of the direct problem (1)-(2) is guaranteed by the following proposition:

Proposition 1 (Theorem 4.2 in [1]) Under regularity assumptions on the coefficients $(\lambda, \mu, \tilde{\lambda}, \tilde{\mu})$, if $\bar{u}_{0} \in H_{0}^{1}(\Omega)^{3}, \bar{u}_{1} \in L^{2}(\Omega)^{3}$ and $f \in L^{\infty}\left(0,+\infty ; L^{2}(\Omega)^{3}\right) \cap$ $L^{2}\left(0,+\infty ; L^{2}(\Omega)^{3}\right)$, then there exists a unique weak solution $u \in L^{\infty}\left(0,+\infty ; H_{0}^{1}(\Omega)^{3}\right) \cap$ $W^{1, \infty}\left(0,+\infty ; L^{2}(\Omega)^{3}\right) \cap W^{2, \infty}\left(0,+\infty ; H^{-1}(\Omega)^{3}\right)$ to the problem (1)-(2).

If we assume now that the coefficient $\tilde{\mu}$ can be decomposed as follows:

$$
\tilde{\mu}(x, t)=p(x) h(t), \quad \forall(x, t) \in \Omega \times(0,+\infty) .
$$

Then, the inverse problem we are interested in is formulated accordingly:

Definition 1 (Inverse problem) Given $\left(\lambda, \mu, \tilde{\lambda}, h, u_{0}, u_{1}, f\right)$, recover $p(x)$, for all $x \in \Omega$, from measurements of

$$
u(x, t), \quad \forall(x, t) \in \Gamma \times(0, T),
$$

where $\Gamma$ is a part of $\partial \Omega$ and $T>0$.

Several authors have dealt with the problem of recovering the coefficients of the viscoelasticity system (1)-(2). Some of them, e.g. Grasselli [2], Janno et al. [3], von Wolfersdorf [4], Cavaterra et al. [5], recovered the time dependance $h$ of the coefficient $\tilde{\mu}$ by reducing the problem to a non-linear Volterra integral equation by using Fourier's method to solve the direct problem and by applying the contraction principle. Others, e.g. Lorenzi [6], Lorenzi and Romanov [7], recovered the space dependance $p$ of the coefficient $\tilde{\mu}$ by using the method of Bukhgeim and Klibanov [8] based on Carleman estimates [9]. Thus, in 2007, Lorenzi et al. [10] recovered the coefficient $p$ from three measurements, relying on the assumption that all the coefficients in the operator (2) are independent of the third space variable. Here, unlike the latter, we recover $p$ from a unique measurement, assuming that it is known in a neighborhood of the boundary. Furthermore, Theorem 1 establishes the logarithmic stability with respect to a unique measurement on an arbitrary part of the boundary for the inverse problem of recovering the coefficient $p$. Its proof is given in Section 2. It relies on a Carleman estimate (Theorem 2) and a sharp unique continuation result (Theorem 3) for the operator (2). We prove theses results in Section 3 and 4 respectively. In Section 5, we propose an adaptive spectral method to approximate the solution of the inverse problem numerically.

\subsection{Stability estimate}

Inverse problems are ill-posed in the classical sense [11]. Stability estimates play thus a special role in the theory. Bukhgeim and Klibanov [8] developed a remarkable method based on Carleman estimates [9] to prove the uniqueness and stability for inverse problems associated to partial differential equations. In [12], using this method, we proved a Hölder stability result with a unique internal measurement for the recovery of $p$ in the system (1)-(3). In this article, we extend this result using a unique continuation estimate and obtain a logarithmic stability result. Although this estimate is weaker, it is nonetheless related to a measurement on an arbitrary part of the boundary. A similar result was proved in Bellassoued [13] for the hyperbolic scalar equation and in Bellassoued et al. [14] for the Lamé system. We follow their method but adapt it to take into account the additive integral term of operator (2). Thus, our 
result can be seen as an extension of the paper [14]. Nevertheless, the presence of the integral term modifies the structure of the equation. Firstly, the latter is not reversible anymore because of the energy dissipation associated with the viscous phenomenon. In practice, this prevents the extension of the solution to $[-T, 0]$ as usually performed. This explains why we need using a point-wise Carleman estimate (see Section 1.2). Secondly, the coefficients of the equation now depend on time and not only on space as for the Lamé system. This implies that the FBI transform classically used to prove the unique continuation result (see Section 1.3) is useless. Hence, we have to introduce a new transformation in order to deal with convolution terms. We need now to define the following condition on a scalar function $q$ :

Condition 1 The scalar function $q$ is said to satisfy Condition 1 if

(i) there exists $K>0$ such that $\forall x \in \bar{\Omega}, q(x) \geq K$,

(ii) there exists $x_{0} \in \mathbb{R}^{3} \backslash \bar{\Omega}$ such that $\forall x \in \bar{\Omega}, \frac{1}{2} q(x)-\nabla q(x) \cdot\left(x-x_{0}\right) \geq 0$.

We can now state the main result:

Theorem 1 (Logarithmic stability) Let $u$ (respectively $\bar{u}$ ) be the solution of the system (1)-(3), associated to the coefficient $p$ (respectively $\bar{p}$ ). We assume that

(H1) $(\lambda, \mu) \in C^{2}(\bar{\Omega})^{2}$ and $(\tilde{\lambda}, \tilde{\mu}) \in C^{2}(\overline{\Omega \times(0,+\infty)})^{2}$ are such that the solutions $u$ and $\bar{u} \in W^{8, \infty}(\Omega \times(0,+\infty))^{3}$,

(H2) $\mu$ and $\lambda+2 \mu$ satisfy Condition 1 with a same $x_{0}$,

(H3) $p=\bar{p}$ is known in a neighborhood $\omega$ of $\partial \Omega$,

(H4) $h(0) \neq 0, h^{\prime}(0)=0$,

(H5) there exists $M>0$ such that, $\forall x \in \overline{\Omega \backslash \omega},\left|\left(\nabla \bar{u}_{0}+\nabla \bar{u}_{0}^{T}\right)(x) \cdot\left(x-x_{0}\right)\right| \geq M$.

Then, for $\Gamma \subset \partial \Omega$ arbitrarily small, there exist $\kappa \in(0,1)$ and $T_{0}>0$ such that, for all $T>T_{0}$, the following estimate holds:

$$
\|p-\bar{p}\|_{H^{2}(\Omega)} \leq C\left[\log \left(2+\frac{C}{\sum_{1 \leq|\alpha| \leq 2}\left\|\partial_{x}^{\alpha}(u-\bar{u})\right\|_{L^{2}(\Gamma \times(0, T))}^{2}}\right)\right]^{-\kappa}
$$

where $C>0$ depends on the $C^{2}(\bar{\Omega})$-norm of $p$ and $\bar{p}$ and on the $W^{8, \infty}(\Omega \times(0, T))^{3}$ norm of $u$ and $\bar{u}$.

An example of an initial datum $\bar{u}_{0}$ verifying $(H 5)$ corresponds to the choice $\bar{u}_{0} \in$ $H_{0}^{1}(\Omega)^{3}$ such that $\bar{u}_{0}(x)=x, \forall x \in \overline{\Omega \backslash \omega}$. Thus,

$$
\left|\left(\nabla \bar{u}_{0}+\nabla \bar{u}_{0}^{T}\right)(x) \cdot\left(x-x_{0}\right)\right|=\left|x-x_{0}\right| \geq d_{0}>0, \quad \forall x \in \overline{\Omega \backslash \omega} .
$$

From Theorem 1, we immediately deduce the Corollary 1.

Corollary 1 (Uniqueness) Under all the hypothesis of Theorem 1, we have then

$$
\begin{aligned}
& \partial_{x}^{\alpha} u(x, t)=\partial_{x}^{\alpha} \bar{u}(x, t), \quad \forall(x, t) \in \Gamma \times(0, T), \quad|\alpha|=1,2 \\
& \Longrightarrow p(x)=\bar{p}(x), \quad \forall x \in \Omega .
\end{aligned}
$$




\subsection{A Carleman estimate}

In a pioneering work [9], Carleman introduced what is now commonly known as a Carleman estimate, in the context of proving the uniqueness in the Cauchy elliptic problem. Since then, the theory of Carleman estimates has been extensively studied. As for a general treatment of Carleman estimates, see Hörmander [15], Isakov [16], Tataru [17], Imanuvilov and Yamamoto [18]. In 2006, Cavaterra et al. [19] modified the point-wise Carleman inequality of Klibanov and Timonov [20] for a hyperbolic scalar equation and integrated, thanks to a change of variable, the integral term of (2). In 2008, Imanuvilov and Yamamoto [21] proved a Carleman estimate for the 3D Lamé system - operator (2) without the integral term. Their approach consisted in decoupling the system by writing the equations satisfied by $u, \nabla \wedge u$ and $\nabla \cdot u$ in view of applying well known results for hyperbolic scalar equations [22]. In this article, we prove a Carleman estimate for the complete integro-differential operator (2) by combination of these two techniques. We start by introducing the following Carleman weight function:

Definition 2 (a Carleman weight function) For $x_{0} \in \mathbb{R}^{3} \backslash \bar{\Omega}$ and $\beta>0$, we introduce a function $\varphi$ in the following way:

$$
\varphi(x, t)=\left|x-x_{0}\right|^{2}-\beta t^{2}, \quad \forall(x, t) \in \Omega \times(0,+\infty) .
$$
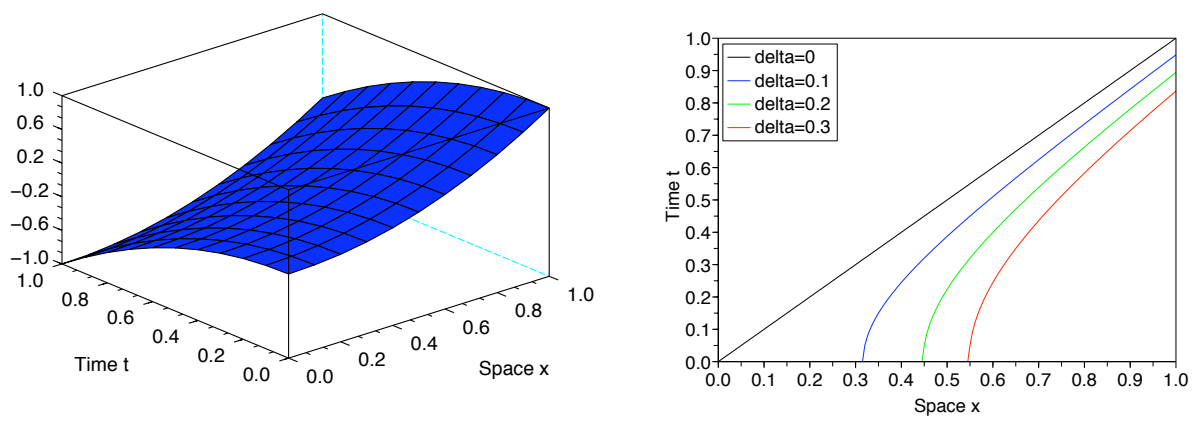

Figure 1. The Carleman weight function $\varphi(x, t)$ (left) and its isovalues $\varphi(x, t)=\delta$ (right) for $x_{0}=0$ and $\beta=1$.

Let us introduce now some notations used in the sequel:

- Distances (see Figure 2)

For $x_{0} \in \mathbb{R}^{3} \backslash \bar{\Omega}$, we note

$$
d_{0}=\inf _{x \in \Omega}\left|x-x_{0}\right| \quad \text { and } \quad d=\sup _{x \in \Omega}\left|x-x_{0}\right|^{2} .
$$

- Domains (see Figure 3)

For $\varepsilon>0$ and $\delta>0$, we note

$$
\begin{aligned}
& \Omega(\varepsilon)=\{x \in \Omega, \operatorname{dist}(x, \partial \Omega)>\varepsilon\}, \quad \Omega(0)=\Omega, \\
& Q(\varepsilon, \delta)=\{(x, t) \in \Omega(\varepsilon) \times(0,+\infty), \varphi(x, t)>\delta\}, \quad Q(0,0)=Q .
\end{aligned}
$$

We notice that $Q \subset \Omega \times(0, T)$ if $T>\frac{d}{\sqrt{\beta}}$. 


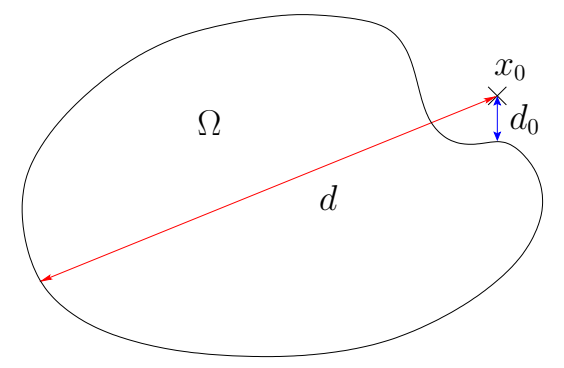

Figure 2. Definitions of the distances $d_{0}$ and $d$.
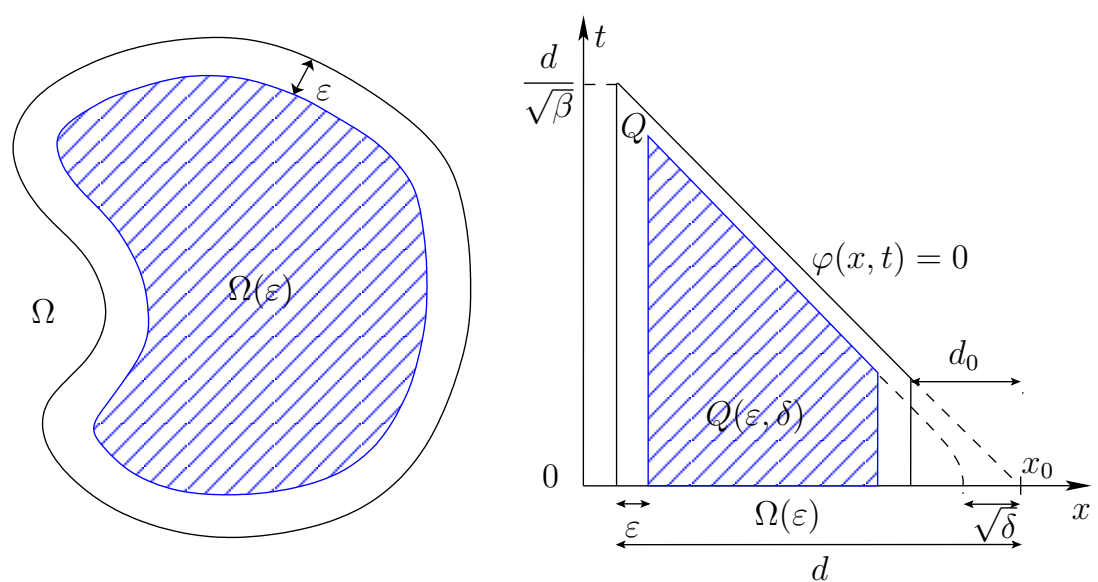

Figure 3. Definitions of the domains $\Omega(\varepsilon)$ and $Q(\varepsilon, \delta)$.

- Norms

For $Q \subset \mathbb{R}^{3} \times \mathbb{R}, \sigma>0$ and $k \in \mathbb{N}$, we introduce the following norms:

$$
\begin{aligned}
\|\star\|_{H^{1, \sigma}(Q)}^{2} & =\left\|\nabla_{x, t} \star\right\|_{L^{2}(Q)}^{2}+\sigma^{2}\|\star\|_{L^{2}(Q)}^{2}, \\
\|\star\|_{H_{x}^{k, \sigma}(Q)}^{2} & =\sum_{|\alpha| \leq k} \sigma^{2(k-|\alpha|)}\left\|\partial_{x}^{\alpha} \star\right\|_{L^{2}(Q)}^{2} .
\end{aligned}
$$

- Constants

$C$ and $C_{j}, 1 \leq j \leq 6$ are generic positive constants.

Equipped with these definitions, we can now state the following result:

Theorem 2 (Carleman estimate) Let $\mathcal{P}$ be the operator defined by (1). We assume that

(H1) $(\lambda, \mu) \in C^{2}(\bar{\Omega})^{2}$ and $(\tilde{\lambda}, \tilde{\mu}) \in C^{2}(\overline{\Omega \times(0,+\infty)})^{2}$,

(H2) $\mu$ and $\lambda+2 \mu$ satisfy Condition 1 with a same $x_{0}$.

Then, there exist $\beta_{0}>0$ and $\sigma_{0}>0$ such that, for all $\beta \leq \beta_{0}$, for all $\sigma \geq \sigma_{0}$, for all $\varepsilon>0$, for all $0<\delta<d_{0}^{2}$ and for all $u \in H^{2}(Q)^{3}$ satisfying $u(x, 0)=0$ or 
$\partial_{t} u(x, 0)=0, \forall x \in \Omega$, the following estimate holds:

$$
\begin{aligned}
\frac{C}{\sigma}\left\|u e^{\sigma \varphi}\right\|_{H_{x}^{2}, \sigma}^{2}(Q(\varepsilon, \delta)) \leq & \left\|(\mathcal{P} u) e^{\sigma \varphi}\right\|_{L^{2}(Q)}^{2}+\left\|\nabla(\mathcal{P} u) e^{\sigma \varphi}\right\|_{L^{2}(Q)}^{2} \\
& +e^{C \sigma}\|u\|_{H^{2}(Q(\varepsilon, \delta) \backslash Q(2 \varepsilon, \delta))}^{2}+\sigma^{3} e^{2 \sigma \delta}\|u\|_{H^{2}(Q)}^{2},
\end{aligned}
$$

where $\varphi$ is defined by (4) and $C>0$ depends on the $C^{2}(\bar{Q})$-norm of the coefficient $\tilde{\mu}$ but is independent of $\sigma$.

The last term of this estimate is rather uncommon since it is global but it will disappear thanks to a clever choice of $\sigma$.

\subsection{A unique continuation result}

In [23], Bellassoued proved a sharp unique continuation result for the Lamé system. He applied a method initially developed by Robbiano [24] which uses the FourierBros-Iagolnitzer (FBI) transform [25] to change the problem near the boundary into a problem for which elliptic estimates can be applied. In this article, we show a similar result for the system (1)-(2) by adapting these techniques. In particular, we propose a new transformation inspired from the FBI transform but which is able to deal with the additive convolution term of the operator (2). The result we prove is the following:

Theorem 3 (Unique continuation) Let $u$ be the vector solution of

$$
\begin{cases}\mathcal{P} u(x, t)=R(x, t), & \forall(x, t) \in \Omega \times(0,+\infty), \\ u(x, 0)=0, & \forall x \in \Omega, \\ \partial_{t} u(x, 0)=0, & \forall x \in \Omega, \\ u(x, t)=0, & \forall(x, t) \in \partial \Omega \times(0,+\infty) .\end{cases}
$$

We assume that

(H1) $(\lambda, \mu) \in C^{2}(\bar{\Omega})^{2}$ and $(\tilde{\lambda}, \tilde{\mu}) \in C^{2}(\overline{\Omega \times(0,+\infty)})^{2}$ are such that the solution $u \in W^{4, \infty}(\Omega \times(0,+\infty))^{3}$,

(H6) $R(x, t)=0, \forall(x, t) \in \omega \times(0,+\infty)$ where $\omega$ is a neighborhood of $\partial \Omega$.

Then, for $\Gamma \subset \partial \Omega$ arbitrarily small, there exists $T_{0}>0$ such that, for all $T \geq T_{0}$ and for all $\rho>0$, the following estimate holds:

$$
\|u\|_{H^{2}\left(\omega \times\left(0, \frac{T}{2}-\rho\right)\right)}^{2} \leq C\left[\log \left(2+\frac{C}{\sum_{1 \leq|\alpha| \leq 2}\left\|\partial_{x}^{\alpha} u\right\|_{L^{2}(\Gamma \times(0,3 T))}^{2}}\right)\right]^{-1}
$$

where $C>0$ depends on the $C^{2}(\overline{\Omega \times(0, T)})$-norm of the coefficient $\tilde{\mu}$ and on the norm $W^{4, \infty}(\Omega \times(0, T))^{3}$ of $u$.

\subsection{Numerical results}

Section 5 presents a numerical example to illustrate one of our theoretical result [12]. Here, we propose a non-quadratic functional to solve the inverse problem. Moreover, as regularization method [26], we use a spectral basis, adapted in space and in frequency to the solution. This idea, combined with mesh adaptation, allows to improve the accuracy of the method by minimizing the numerical error. The unknown parameter is successfully recovered at each vertex of the discretized domain. 
Logarithmic stability in determination of a $3 D$ viscoelastic coefficient

\section{Proof of Theorem 1}

This section presents the proof of the stability result (Theorem 1). The idea of the proof is the following. Firstly, we bring the unknown parameter $p$ to the source by writing the equation satisfied by $\hat{v}=\partial_{t}(u-\bar{u})$. Then, we use the method of Bukhgeim and Klibanov, i.e. we differentiate the previous equation to bring the parameter in the initial condition and we apply the Carleman estimate of Theorem 2 to the new variable $\partial_{t} \hat{v}$ in order to bound the initial energy. Thanks to a Carleman estimate for a first order operator (Lemma 2), we come back to the coefficient in the estimate. Thus, we obtain a first Hölder stability result with an internal measurement on a neighborhood of the boundary. Finally, we combine this result with the unique continuation relation of Theorem 3 to conclude.

Let $u$ (respectively $\bar{u}$ ) be the solution of the system (1)-(3), associated with the coefficient $p$ (respectively $\bar{p}$ ). We suppose that hypothesis (H1)-(H5) hold and we fix $\Gamma \subset \partial \Omega$ arbitrarily small. By linearity and without loss of generality, we can suppose that $\bar{u}_{1}=0$.

\subsection{Bring the unknown parameter $p$ to the source}

We suppose $(\bar{p}, \bar{u})$ known and we introduce $\hat{p}=p-\bar{p}$ and $\hat{u}=u-\bar{u}$ which satisfies the following equation:

$$
\begin{aligned}
& \mathcal{P} \hat{u}(x, t)=\partial_{t}^{2} \hat{u}(x, t)-\nabla \cdot\left(\mu(x)\left(\nabla \hat{u}(x, t)+\nabla \hat{u}(x, t)^{T}\right)+\lambda(x)(\nabla \cdot \hat{u})(x, t) I\right) \\
& +\int_{0}^{t} \nabla \cdot\left(h(s) p(x)\left(\nabla \hat{u}(x, t-s)+\nabla \hat{u}(x, t-s)^{T}\right)+\tilde{\lambda}(x, s)(\nabla \cdot \hat{u})(x, t-s) I\right) d s \\
& =-\int_{0}^{t} h(s) \nabla \cdot\left(\hat{p}(x)\left(\nabla \bar{u}(x, t-s)+\nabla \bar{u}(x, t-s)^{T}\right)\right) d s, \quad \forall(x, t) \in \Omega \times(0,+\infty),
\end{aligned}
$$

with null initial and boundary conditions. We notice that the coefficient $\hat{p}$ we wish to recover appears in the source term. The problem is that this source term vanishes at the initial time $t=0$, so it does not satisfy the hypothesis of the method of Bukhgeim and Klibanov [8]. To overcome this problem, we derive the equation in time and set

$$
\hat{v}(x, t)=\partial_{t} \hat{u}(x, t), \quad \forall(x, t) \in \Omega \times(0,+\infty) .
$$

Then, $\hat{v}$ satisfies the equation

$$
\begin{aligned}
\mathcal{P} \hat{v}(x, t)= & -\int_{0}^{t} h(s) \nabla \cdot\left(\hat{p}(x)\left(\nabla \bar{v}(x, t-s)+\nabla \bar{v}(x, t-s)^{T}\right)\right) d s \\
& -h(t) \nabla \cdot\left(\hat{p}(x)\left(\nabla \bar{u}_{0}(x)+\nabla \bar{u}_{0}(x)^{T}\right)\right), \quad \forall(x, t) \in \Omega \times(0,+\infty),
\end{aligned}
$$

with null initial and boundary conditions. As a consequence, the unknown coefficient is still in the source term but it is not vanishing at the initial time anymore.

\subsection{Use the method of Bukhgeim and Klibanov}

We derive (5) and set

$$
\hat{w}(x, t)=\partial_{t} \hat{v}(x, t)=\partial_{t}^{2} \hat{u}(x, t), \quad \forall(x, t) \in \Omega \times(0,+\infty),
$$

which satisfies then

$$
\begin{aligned}
\mathcal{P} \hat{w}(x, t)= & -\int_{0}^{t} h(s) \nabla \cdot\left(\hat{p}(x)\left(\nabla \bar{w}(x, t-s)+\nabla \bar{w}(x, t-s)^{T}\right)\right) d s \\
& -h^{\prime}(t) \nabla \cdot\left(\hat{p}(x)\left(\nabla \bar{u}_{0}(x)+\nabla \bar{u}_{0}(x)^{T}\right)\right), \quad \forall(x, t) \in \Omega \times(0,+\infty),
\end{aligned}
$$


with the following initial and boundary conditions:

$$
\left\{\begin{array}{lr}
\hat{w}(x, 0)=0, & \forall x \in \Omega, \\
\partial_{t} \hat{w}(x, 0)=-h(0) \nabla \cdot\left(\hat{p}(x)\left(\nabla \bar{u}_{0}(x)+\nabla \bar{u}_{0}(x)^{T}\right)\right), \\
\hat{w}(x, t)=0, & \forall(x, t) \in \partial \Omega \times(0,+\infty) .
\end{array}\right.
$$

Therefore, the coefficient $\hat{p}$ that we want to recover appears now in the initial conditions. We are going to write an inequality by bounding the energy of the system (7) by the source and the observations. To do that, we apply the Carleman estimate of Theorem 2 to $\hat{w}$ and to its time derivatives.

\subsection{Apply the Carleman estimate}

Theorem 2 states that for $\varphi$ defined by equation (4) with $\beta$ sufficiently small, for $\sigma$ sufficiently large, for $\varepsilon>0$ and $0<\delta<d_{0}^{2}$, the following estimate holds:

$$
\begin{aligned}
& \frac{1}{\sigma}\left\|\hat{w} e^{\sigma \varphi}\right\|_{H_{x}^{2, \sigma}(Q(\varepsilon, \delta))}^{2} \leq C\left(e^{C \sigma}\|\hat{w}\|_{H^{2}(Q(\varepsilon, \delta) \backslash Q(2 \varepsilon, \delta))}^{2}+\sigma^{3} e^{2 \sigma \delta}\|\hat{w}\|_{H^{2}(Q)}^{2}\right. \\
& +\int_{Q}\left|\int_{0}^{t} h(s) \nabla \cdot\left(\hat{p}(x)\left(\nabla \bar{w}(x, t-s)+\nabla \bar{w}(x, t-s)^{T}\right)\right) d s\right|^{2} e^{2 \sigma \varphi(x, t)} d x d t \\
& +\int_{Q}\left|\int_{0}^{t} h(s) \nabla\left(\nabla \cdot\left(\hat{p}(x)\left(\nabla \bar{w}(x, t-s)+\nabla \bar{w}(x, t-s)^{T}\right)\right)\right) d s\right|^{2} e^{2 \sigma \varphi(x, t)} d x d t \\
& \left.+\left\|h^{\prime} \nabla \cdot\left(\hat{p}\left(\nabla \bar{u}_{0}+\nabla \bar{u}_{0}^{T}\right)\right) e^{\sigma \varphi}\right\|_{L^{2}(Q)}^{2}+\left\|h^{\prime} \nabla\left(\nabla \cdot\left(\hat{p}\left(\nabla \bar{u}_{0}+\nabla \bar{u}_{0}^{T}\right)\right)\right) e^{\sigma \varphi}\right\|_{L^{2}(Q)}^{2}\right) .
\end{aligned}
$$

We now need a result to bound the integral terms. This is given by the following lemma:

Lemma 1 (Lemma 3.1.1 in [20]) Let $\varphi$ be defined by (4). There exists $C>0$ such that, for all $\sigma>0$ and $u \in L^{2}(Q)$,

$$
\int_{Q}\left(\int_{0}^{t}|u(x, s)| d s\right)^{2} e^{2 \sigma \varphi(x, t)} d x d t \leq \frac{C}{\sigma} \int_{Q}|u(x, t)|^{2} e^{2 \sigma \varphi(x, t)} d x d t .
$$

We use the result of Lemma 1 and the fact that $(\hat{p}, h)$ is bounded in $\Omega \times(0,+\infty)$ (H1) to write:

$$
\begin{aligned}
& \frac{C}{\sigma}\left\|\hat{w} e^{\sigma \varphi}\right\|_{H_{x}^{2, \sigma}(Q(\varepsilon, \delta))}^{2} \leq e^{C \sigma}\|\hat{w}\|_{H^{2}(Q(\varepsilon, \delta) \backslash Q(2 \varepsilon, \delta))}^{2}+\sigma^{3} e^{2 \sigma \delta}\|\hat{w}\|_{H^{2}(Q)}^{2} \\
& \quad+\left\|\nabla \cdot\left(\hat{p}\left(\nabla \bar{u}_{0}+\nabla \bar{u}_{0}^{T}\right)\right) e^{\sigma \varphi}\right\|_{L^{2}(Q)}^{2}+\left\|\nabla\left(\nabla \cdot\left(\hat{p}\left(\nabla \bar{u}_{0}+\nabla \bar{u}_{0}^{T}\right)\right)\right) e^{\sigma \varphi}\right\|_{L^{2}(Q)}^{2} .
\end{aligned}
$$

Thus, taking into account the fact that $\bar{u}_{0} \in W^{8, \infty}(\overline{\Omega \times(0,+\infty)})$ (H1), we obtain

$$
\begin{aligned}
\frac{C}{\sigma}\left\|\hat{w} e^{\sigma \varphi}\right\|_{H_{x}^{2, \sigma}(Q(\varepsilon, \delta))}^{2} \leq & e^{C \sigma}\left\|\partial_{t}^{2} \hat{u}\right\|_{H^{2}(Q(\varepsilon, \delta) \backslash Q(2 \varepsilon, \delta))}^{2}+\sigma^{3} e^{2 \sigma \delta}\left\|\partial_{t}^{2} \hat{u}\right\|_{H^{2}(Q)}^{2} \\
& +\sum_{|\alpha| \leq 2}\left\|\left(\partial_{x}^{\alpha} \hat{p}\right) e^{\sigma \varphi}\right\|_{L^{2}(Q)}^{2} .
\end{aligned}
$$

Similarly, we obtain, for $i=1,2$,

$$
\begin{aligned}
\frac{C}{\sigma}\left\|\left(\partial_{t}^{i} \hat{w}\right) e^{\sigma \varphi}\right\|_{H_{x}^{2, \sigma}(Q(\varepsilon, \delta))}^{2} & \leq e^{C \sigma}\left\|\partial_{t}^{2+i} \hat{u}\right\|_{H^{2}(Q(\varepsilon, \delta) \backslash Q(2 \varepsilon, \delta))}^{2}+\sigma^{3} e^{2 \sigma \delta}\left\|\partial_{t}^{2+i} \hat{u}\right\|_{H^{2}(Q)}^{2} \\
+ & \sum_{|\alpha| \leq 2}\left\|\left(\partial_{x}^{\alpha} \hat{p}\right) e^{\sigma \varphi}\right\|_{L^{2}(Q)}^{2} .
\end{aligned}
$$

In order to apply the Carleman estimate, we verified that the functions $\partial_{t} \hat{w}$ and $\partial_{t}^{2} \hat{w}$ have one of their initial conditions null. This requirement is true since we supposed $h^{\prime}(0)=0$ in $(H 4)$. We will now bound the initial energy of the system (7). 


\subsection{Bound the initial energy}

We define the cut-off function $\chi_{1} \in C^{\infty}\left(\mathbb{R}^{3} \times \mathbb{R}\right)$ by $0 \leq \chi_{1} \leq 1$ and

$$
\chi_{1}(x, t)= \begin{cases}1, & \text { if } \quad(x, t) \in Q(0,2 \delta), \\ 0, & \text { if } \quad(x, t) \in Q \backslash \overline{Q(0, \delta)},\end{cases}
$$

and we set

$$
w^{*}(x, t)=\chi_{1}(x, t) \hat{w}(x, t), \quad \forall(x, t) \in \Omega \times(0,+\infty) .
$$

This function is illustrated in Figure 4.

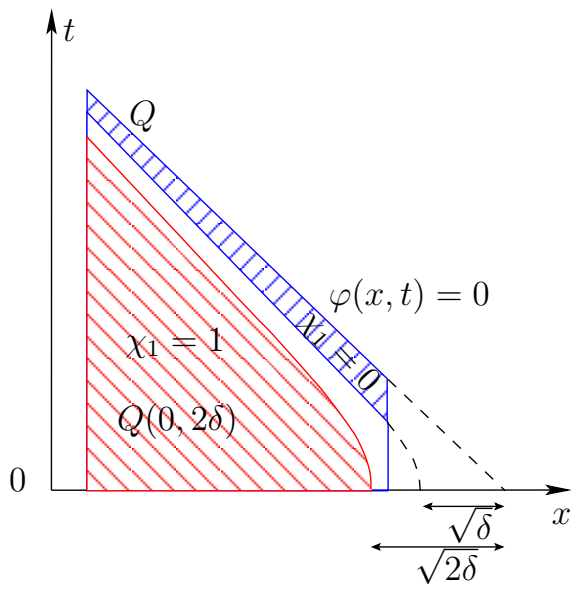

Figure 4. Definition of the cut-off function $\chi_{1}$.

Since the time derivative of $w^{*}$ is null outside $Q(0, \delta)$, i.e. for

$$
t \geq t_{x}^{\delta}=\sqrt{\frac{\left|x-x_{0}\right|^{2}-\delta}{\beta}}
$$

which is well defined for all $x \in \Omega$ because $\delta<d_{0}^{2}$, we can write:

$$
\begin{aligned}
& \int_{\Omega(\varepsilon)}\left|\partial_{t} w^{*}(x, 0)\right|^{2} e^{2 \sigma \varphi(x, 0)} d x=-\int_{\Omega(\varepsilon)} \int_{0}^{t_{x}^{\delta}} \partial_{t}\left(\left|\partial_{t} w^{*}(x, t)\right|^{2} e^{2 \sigma \varphi(x, t)}\right) d x d t \\
& =\int_{Q(\varepsilon, \delta)}\left(4 \beta t \sigma\left|\partial_{t} w^{*}(x, t)\right|^{2} e^{2 \sigma \varphi(x, t)}-2 \partial_{t} w^{*}(x, t) \cdot \partial_{t}^{2} w^{*}(x, t) e^{2 \sigma \varphi(x, t)}\right) d x d t .
\end{aligned}
$$

In addition, we have

$$
\begin{aligned}
& \partial_{t} w^{*}(x, t)=\chi_{1}(x, t) \partial_{t} \hat{w}(x, t)+\partial_{t} \chi_{1}(x, t) \hat{w}(x, t), \quad \forall(x, t) \in \Omega \times(0,+\infty), \\
& \partial_{t}^{2} w^{*}(x, t)=\chi_{1}(x, t) \partial_{t}^{2} \hat{w}(x, t)+2 \partial_{t} \chi_{1}(x, t) \partial_{t} \hat{w}(x, t)+\partial_{t}^{2} \chi_{1}(x, t) \hat{w}(x, t) .
\end{aligned}
$$

Therefore, we obtain

$$
\begin{aligned}
\int_{\Omega(\varepsilon)} & \left|\partial_{t} w^{*}(x, 0)\right|^{2} e^{2 \sigma \varphi(x, 0)} d x \\
& \leq C\left(\int_{Q(\varepsilon, \delta)}\left(\sigma|\hat{w}(x, t)|^{2}+\sigma\left|\partial_{t} \hat{w}(x, t)\right|^{2}+\left|\partial_{t}^{2} \hat{w}(x, t)\right|^{2}\right) e^{2 \sigma \varphi(x, t)} d x d t\right) .
\end{aligned}
$$


We do the same work for the derivatives in $x$ for $|\alpha| \leq 2$ and thus we obtain:

$$
\begin{aligned}
& \sum_{|\alpha| \leq 2} \sigma^{2(1-|\alpha|)} \int_{\Omega(\varepsilon)}\left|\partial_{x}^{\alpha}\left(\partial_{t} \hat{w}\right)(x, 0)\right|^{2} e^{2 \sigma \varphi(x, 0)} d x \\
& \quad \leq \frac{C}{\sigma}\left(\left\|\hat{w} e^{\sigma \varphi}\right\|_{H_{x}^{2, \sigma}(Q(\varepsilon, \delta))}^{2}+\left\|\left(\partial_{t} \hat{w}\right) e^{\sigma \varphi}\right\|_{H_{x}^{2, \sigma}(Q(\varepsilon, \delta))}^{2}+\left\|\left(\partial_{t}^{2} \hat{w}\right) e^{\sigma \varphi}\right\|_{H_{x}^{2, \sigma}(Q(\varepsilon, \delta))}^{2}\right) \\
& \quad \leq C\left(\sum_{|\alpha| \leq 2}\left\|\left(\partial_{x}^{\alpha} \hat{p}\right) e^{\sigma \varphi}\right\|_{L^{2}(Q)}^{2}+e^{C \sigma}\|\hat{u}\|_{H^{6}(Q(\varepsilon, 2 \delta) \backslash Q(2 \varepsilon, \delta))}^{2}+\sigma^{3} e^{2 \sigma \delta}\|\hat{u}\|_{H^{6}(Q)}^{2}\right)
\end{aligned}
$$

where we used (9) in the last inequality. We have to go back to the coefficient in the left hand side.

\subsection{Return to the coefficient in the estimate}

To this end, we need a Carleman estimate for a first order operator. It is given by the following lemma:

Lemma 2 (Lemma 3.2 in [18]) We consider the following first order partial differential operator:

$$
\mathcal{R}(x) \star=a(x) \cdot \nabla \star+a_{0}(x) \star, \quad \forall x \in \Omega,
$$

with

$a_{0} \in C^{0}(\bar{\Omega}), \quad\left\|a_{0}\right\|_{C^{2}(\bar{\Omega})} \leq M_{1} \quad$ and $\quad a \in C^{1}(\bar{\Omega})^{n}, \quad\|a\|_{C^{2}(\bar{\Omega})} \leq M_{2}$,

$\exists x_{0}, \quad\left|a(x) \cdot\left(x-x_{0}\right)\right| \geq M_{3}>0, \quad \varphi_{0}(x)=\left|x-x_{0}\right|^{2}, \quad \forall x \in \bar{\Omega}$.

Then, there exist $\sigma_{0}>0$ and $C>0$ such that, for all $\sigma \geq \sigma_{0}$ and for all $q \in C_{0}^{2}(\Omega)$,

$\sigma^{2} \sum_{|\alpha| \leq 2} \int_{\Omega}\left|\partial_{x}^{\alpha} q(x)\right|^{2} e^{2 \sigma \varphi_{0}(x)} d x \leq C \sum_{|\alpha| \leq 2} \int_{\Omega}\left|\partial_{x}^{\alpha}(\mathcal{R}(x) q(x))\right|^{2} e^{2 \sigma \varphi_{0}(x)} d x$.

Since the coefficient $\hat{p}$ satisfies the following first order system:

$$
h(0) \nabla \cdot\left(\hat{p}(x)\left(\nabla \bar{u}_{0}(x)+\nabla \bar{u}_{0}(x)^{T}\right)\right)=-\partial_{t} w^{*}(x, 0),
$$

we can apply Lemma 2 to each equation of the system with

$q(x)=\hat{p}(x), \quad \mathcal{R}(x) q(x)=-\partial_{t} w^{*}(x, 0), \quad \varphi_{0}(x)=\varphi(x, 0)$,

$a_{0}(x)=h(0) \nabla \cdot\left(\nabla \bar{u}_{0}(x)+\nabla \bar{u}_{0}(x)^{T}\right), \quad a(x)=h(0)\left(\nabla \bar{u}_{0}(x)+\nabla \bar{u}_{0}(x)^{T}\right)$,

which satisfy (H5) and we obtain then

$\sum_{|\alpha| \leq 2} \int_{\Omega(\varepsilon)}\left|\partial_{x}^{\alpha} \hat{p}(x)\right|^{2} e^{2 \sigma \varphi(x, 0)} d x \leq C \sum_{|\alpha| \leq 2} \sigma^{2(1-|\alpha|)} \int_{\Omega(\varepsilon)}\left|\partial_{x}^{\alpha}\left(\partial_{t} w^{*}\right)(x, 0)\right|^{2} e^{2 \sigma \varphi(x, 0)} d x$.

We supposed in (H3) that we know $p$ in a neighborhood $\omega$ of the boundary $\partial \Omega$, i.e. $\hat{p}=0$ in $\omega$ and so we can assume $\varepsilon$ to be sufficiently small such that $\Omega \backslash \Omega(\varepsilon) \subset \omega$. This allows to integrate on $\Omega$ in the left hand side. Then, we have

$$
\begin{aligned}
& \sum_{|\alpha| \leq 2} \int_{\Omega}\left|\partial_{x}^{\alpha} \hat{p}(x)\right|^{2} e^{2 \sigma \varphi(x, 0)} d x \\
& \leq C\left(\sum_{|\alpha| \leq 2}\left\|\left(\partial_{x}^{\alpha} \hat{p}\right) e^{\sigma \varphi}\right\|_{L^{2}(Q)}^{2}+e^{C \sigma}\|\hat{u}\|_{H^{6}(Q(\varepsilon, \delta) \backslash Q(2 \varepsilon, \delta))}^{2}+\sigma^{3} e^{2 \sigma \delta}\|\hat{u}\|_{H^{6}(Q)}^{2}\right) .
\end{aligned}
$$


We can absorb the first term of the right hand side of (10) in the left hand side, thanks to the Carleman weights. Indeed,

$$
\sum_{|\alpha| \leq 2}\left\|\left(\partial_{x}^{\alpha} \hat{p}\right) e^{\sigma \varphi}\right\|_{L^{2}(Q)}^{2}=\sum_{|\alpha| \leq 2} \int_{\Omega}\left|\partial_{x}^{\alpha} \hat{p}(x)\right|^{2} e^{2 \sigma \varphi(x, 0)}\left(\int_{0}^{t_{x}^{0}} e^{2 \sigma(\varphi(x, t)-\varphi(x, 0))} d t\right) d x
$$

with, $\forall x \in \Omega$ :

$$
\int_{0}^{t_{x}^{0}} e^{2 \sigma(\varphi(x, t)-\varphi(x, 0))} d t \leq \int_{0}^{+\infty} e^{-2 \sigma \beta t^{2}} d t=\frac{1}{\sqrt{2 \sigma \beta}} \int_{0}^{+\infty} e^{-z^{2}} d z=\frac{C}{\sqrt{\sigma}} .
$$

Then,

$$
\sum_{|\alpha| \leq 2}\left\|\left(\partial_{x}^{\alpha} \hat{p}\right) e^{\sigma \varphi}\right\|_{L^{2}(Q)}^{2} \leq \frac{C}{\sqrt{\sigma}} \sum_{|\alpha| \leq 2} \int_{\Omega}\left|\partial_{x}^{\alpha} \hat{p}(x)\right|^{2} e^{2 \sigma \varphi(x, 0)} d x .
$$

Finally, we obtain

$$
\begin{aligned}
e^{2 \sigma d_{0}^{2}}\|\hat{p}\|_{H^{2}(\Omega)}^{2} & \leq \sum_{|\alpha| \leq 2} \int_{\Omega}\left|\partial_{x}^{\alpha} \hat{p}(x)\right|^{2} e^{2 \sigma \varphi(x, 0)} d x \\
& \leq C\left(e^{C \sigma}\|\hat{u}\|_{H^{6}(Q(\varepsilon, \delta) \backslash Q(2 \varepsilon, \delta))}^{2}+\sigma^{3} e^{2 \sigma \delta}\|\hat{u}\|_{H^{6}(Q)}^{2}\right),
\end{aligned}
$$

and thus, we conclude that

$$
\|\hat{p}\|_{H^{2}(\Omega)}^{2} \leq C\left(e^{C \sigma}\|\hat{u}\|_{H^{6}(Q(\varepsilon, \delta) \backslash Q(2 \varepsilon, \delta))}^{2}+e^{-\sigma\left(d_{0}^{2}-\delta\right)}\right),
$$

with $l=d_{0}^{2}-\delta>0$, since $\sup _{\sigma}\left(\sigma^{3} e^{-\sigma l}\right)<+\infty$ and since we assumed in (H1) that $\hat{u}$ is in $W^{8, \infty}(Q)$. In order to have

we propose to set

$$
e^{C \sigma}\|\hat{u}\|_{H^{6}(Q(\varepsilon, \delta) \backslash Q(2 \varepsilon, \delta))}^{2}=e^{-\sigma l},
$$

$$
\sigma=-\frac{1}{l+C} \log \left(\|\hat{u}\|_{H^{6}(Q(\varepsilon, \delta) \backslash Q(2 \varepsilon, \delta))}^{2}\right) .
$$

We have $\sigma>0$ if we suppose that $\|\hat{u}\|_{H^{6}(Q(\varepsilon, \delta) \backslash Q(2 \varepsilon, \delta))}^{2}<1$. Thus, the right hand side of the equation can be written as follows:

$$
e^{C \sigma}\|\hat{u}\|_{H^{6}(Q(\varepsilon, \delta) \backslash Q(2 \varepsilon, \delta))}^{2}+e^{-\sigma l}=2\left(\|\hat{u}\|_{H^{6}(Q(\varepsilon, \delta) \backslash Q(2 \varepsilon, \delta))}^{2}\right)^{\frac{l}{l+C}} .
$$

We use now the following interpolation result:

Lemma 3 (Proposition 4 in [27]) For all $m \in \mathbb{N}^{*}$, there exists a constant $C>0$ such that, for all $r$ satisfying $0 \leq r \leq m$ and for all $u \in H^{m}(Q)$, we have

$$
\|u\|_{H^{r}(Q)} \leq C\|u\|_{L^{2}(Q)}^{1-\frac{r}{m}}\|u\|_{H^{m}(Q)}^{\frac{r}{m}}
$$

We apply Lemma 3 to the second derivative of $\hat{u} \in H^{8}(Q)$ with $m=6$ and $r=2$ in order to write:

$$
\|\hat{u}\|_{H^{6}(Q(\varepsilon, \delta) \backslash Q(2 \varepsilon, \delta))} \leq C\|\hat{u}\|_{H^{2}(Q(\varepsilon, \delta) \backslash Q(2 \varepsilon, \delta))}^{1 / 3}\|\hat{u}\|_{H^{8}(Q(\varepsilon, \delta) \backslash Q(2 \varepsilon, \delta))}^{2 / 3} .
$$

Hence,

$$
\|\hat{p}\|_{H^{2}(\Omega)} \leq C\left(\|\hat{u}\|_{H^{2}(Q(\varepsilon, \delta) \backslash Q(2 \varepsilon, \delta))}^{1 / 3}\right)^{\frac{l}{l+C}} .
$$

So we already have a stability result with observation in $Q(\varepsilon, \delta) \backslash Q(2 \varepsilon, \delta)$ which is included in $(\Omega(\varepsilon) \backslash \Omega(2 \varepsilon)) \times(0, T)$ if $T>\frac{d}{\sqrt{\beta}}$. 


\subsection{Use the unique continuation result}

We can now apply Theorem 3 to $\hat{u}$. We verify that hypothesis (H6) holds, i.e. that

$$
R(x, t)=-\int_{0}^{t} h(s) \nabla \cdot\left(\hat{p}(x)\left(\nabla \bar{u}(x, t-s)+\nabla \bar{u}(x, t-s)^{T}\right)\right) d s
$$

vanishes in $\Omega \backslash \Omega(2 \varepsilon) \subset \omega$ and choose $\rho<\delta$. We deduce that, if $T$ is large enough, we have

$$
\|\hat{u}\|_{H^{2}(Q(\varepsilon, \delta) \backslash Q(2 \varepsilon, \delta))}^{2} \leq C\left[\log \left(2+\frac{C}{\sum_{|\alpha|=1}^{2}\left\|\partial_{x}^{\alpha} \hat{u}\right\|_{L^{2}(\Gamma \times(0,6 T))}^{2}}\right)\right]^{-1} .
$$

Therefore,

$$
\|\hat{p}\|_{H^{2}(\Omega)} \leq C\left[\log \left(2+\frac{C}{\sum_{|\alpha|=1}^{2}\left\|\partial_{x}^{\alpha} \hat{u}\right\|_{L^{2}(\Gamma \times(0,6 T))}^{2}}\right)\right]^{-\frac{l}{6(l+C)}} .
$$

We set $\kappa=\frac{l}{6(l+C)} \in(0,1)$ and change $6 T$ by $T$. This achieves to prove Theorem 1 .

\section{Proof of Theorem 2}

This section is devoted to the proof of the Carleman estimate (Theorem 2). The proof consists in decoupling the system of equations as in [21] by writing the equations satisfied by $u, \nabla \wedge u$ and $\nabla \cdot u$. Then, the idea is to introduce a change of variable as in [19] to reduce the problem to a scalar hyperbolic equation for which the Carleman estimate is well known (Corollary 2). Finally, we come back to the initial variable by a series of inequalities assuming the regularity of the coefficients and using the fundamental Lemma 1.

Let $u$ be the solution of the system

with

$$
\begin{cases}\mathcal{P} u(x, t)=F(x, t), & \forall(x, t) \in \Omega \times(0,+\infty), \\ u(x, 0)=0 \quad \text { or } \quad \partial_{t} u(x, 0)=0, & \forall x \in \Omega, \\ u(x, t)=0, & \forall(x, t) \in \partial \Omega \times(0,+\infty),\end{cases}
$$

$$
\begin{aligned}
& \mathcal{P} u(x, t)=\partial_{t}^{2} u(x, t)-\nabla \cdot\left(\mu(x)\left(\nabla u(x, t)+\nabla u(x, t)^{T}\right)+\lambda(x)(\nabla \cdot u)(x, t) I\right) \\
& \quad+\int_{0}^{t} \nabla \cdot\left(\tilde{\mu}(x, s)\left(\nabla u(x, t-s)+\nabla u(x, t-s)^{T}\right)+\tilde{\lambda}(x, s)(\nabla \cdot u)(x, t-s) I\right) d s .
\end{aligned}
$$

Let assume that hypothesis $(H 1)$ and $(H 2)$ are satisfied. We have first to decouple the equations.

\subsection{Decouple the system of equations}

To this end, we develop the system (13) as follows:

$$
\begin{aligned}
\partial_{t}^{2} u(x, t)-\mu(x) \Delta u(x, t)-(\mu+\lambda)(x) \nabla(\nabla \cdot u)(x, t)-(\nabla \cdot u)(x, t) \nabla \lambda(x) \\
\quad-\left(\nabla u(x, t)+\nabla u(x, t)^{T}\right) \cdot \nabla \mu(x)+\int_{0}^{t} \tilde{\mu}(x, t-s) \Delta u(x, s) d s \\
\quad+\int_{0}^{t}(\nabla \cdot u)(x, s) \nabla \tilde{\lambda}(x, t-s) d s+\int_{0}^{t}(\tilde{\mu}+\tilde{\lambda})(x, t-s) \nabla(\nabla \cdot u)(x, s) d s \\
\quad+\int_{0}^{t}\left(\nabla u(x, s)+\nabla u(x, s)^{T}\right) \cdot \nabla \tilde{\mu}(x, t-s) d s=F(x, t) \quad \forall(x, t) \in \Omega \times(0,+\infty) .
\end{aligned}
$$


Notice the change of variable in the convolution terms. Then, we take the curl and the divergence of this system. We introduce the vectors $u_{1}=u, u_{3}=\nabla \wedge u$ and the scalar $u_{2}=\nabla \cdot u$ which satisfy then the following system of seven equations.

$$
\begin{aligned}
& \partial_{t}^{2} u_{i}(x, t)-q_{i}(x) \Delta u_{i}(x, t)+\int_{0}^{t} \tilde{q}_{i}(x, t-s) \Delta u_{i}(x, s) d s \\
&=F_{i}(x, t)+A_{i}\left(u_{1}, u_{2}, u_{3}\right)(x, t), \quad \forall i \in\{1,2,3\},
\end{aligned}
$$

where we set

$$
\begin{aligned}
& q_{1}=q_{2}=\mu, \quad q_{3}=\lambda+2 \mu, \quad \tilde{q}_{1}=\tilde{q}_{2}=\tilde{\mu}, \quad \tilde{q}_{3}=\tilde{\lambda}+2 \tilde{\mu}, \\
& F_{1}=F, \quad F_{2}=\nabla \wedge F, \quad \text { and } \quad F_{3}=\nabla \cdot F \text {. }
\end{aligned}
$$

Here, the coupling terms $A_{i}$ are first order integro-differential operators in $x$ and $t$, with coefficients bounded in $\Omega \times(0,+\infty)$, according to hypothesis $(H 1)$. These equations are coupled only at order 1 , therefore we can apply the results known for the scalar equations. However, the problem is now that the trace of the functions $\nabla \cdot u$ and $\nabla \wedge u$ on $\partial \Omega$ are not defined anymore. We need now to change the integrodifferential hyperbolic equation (14) into a hyperbolic equation in order to apply the classical results.

\subsection{Use a change of variable}

We introduce the following change of variable:

$\tilde{u}_{i}(x, t)=q_{i}(x) u_{i}(x, t)-\int_{0}^{t} \tilde{q}_{i}(x, t-s) u_{i}(x, s) d s, \quad \forall i \in\{1,2,3\}$.

Then, we have, for all $i \in\{1,2,3\}$ and for all $(x, t) \in \Omega \times(0,+\infty)$,

$$
\begin{array}{r}
\partial_{t}^{2} \tilde{u}_{i}(x, t)=q_{i}(x) \partial_{t}^{2} u_{i}(x, t)-\int_{0}^{t} \partial_{t}^{2} \tilde{q}_{i}(x, t-s) u_{i}(x, s) d s \\
+\partial_{t} \tilde{q}_{i}(x, 0) u_{i}(x, t)+\tilde{q}_{i}(x, 0) \partial_{t} u_{i}(x, t),
\end{array}
$$

and

$$
\begin{aligned}
& \Delta \tilde{u}_{i}(x, t)=q_{i}(x) \Delta u_{i}(x, t)-\int_{0}^{t} \tilde{q}_{i}(x, t-s) \Delta u_{i}(x, s) d s+2 \nabla q_{i}(x) \cdot \nabla u_{i}(x, t) \\
& +\Delta q_{i}(x) u_{i}(x, t)-2 \int_{0}^{t} \nabla \tilde{q}_{i}(x, t-s) \cdot \nabla u_{i}(x, s) d s-\int_{0}^{t} \Delta \tilde{q}_{i}(x, t-s) u_{i}(x, s) d s .
\end{aligned}
$$

Therefore, for all $i \in\{1,2,3\}$ and for all $(x, t) \in \Omega \times(0,+\infty)$, the $\tilde{u}_{i}$ satisfy an hyperbolic system of the type:

$$
\begin{aligned}
\partial_{t}^{2} \tilde{u}_{i}(x, t)-q_{i}(x) \Delta \tilde{u}_{i}(x, t) \\
=q_{i}(x)\left(F_{i}(x, t)+A_{i}\left(u_{1}, u_{2}, u_{3}\right)(x, t)\right)+L_{i}\left(u_{i}\right)(x, t),
\end{aligned}
$$

where the $L_{i}$ are first order integro-differential operators, the coefficients of which are bounded in $\Omega \times(0,+\infty)$. We now need a Carleman estimate for a scalar hyperbolic equation. 


\subsection{Use a Carleman estimate for an hyperbolic scalar equation}

The equations we consider are valid in $(0,+\infty)$ and because of the presence of the integral term, we can not extend the solution to $(-\infty, 0)$. That is why we can not use a classical global Carleman estimate found in the literature. Therefore, we will start with the following point-wise Carleman estimate stated in $Q(0, \delta)$ :

Lemma 4 (Theorem 2.2.4 in [20]) Let $q \in C^{2}(\bar{\Omega})$ satisfy Condition 1 and $\varphi$ be defined by (4). Then, there exist $\beta_{0}>0$ and $\sigma_{0}>0$ such that, for all $\beta \leq \beta_{0}$, for all $\sigma \geq \sigma_{0}$, for all $\delta>0$ and for all $u \in H^{2}(Q(0, \delta))$, we have

$$
\begin{aligned}
\left(\sigma\left|\nabla_{x, t} u(x, t)\right|^{2}+\right. & \left.\sigma^{3}|u(x, t)|^{2}\right) e^{2 \sigma \varphi(x, t)}+\nabla \cdot U(x, t)+\partial_{t} V(x, t) \\
& \leq C\left|\partial_{t}^{2} u(x, t)-q(x) \Delta u(x, t)\right|^{2} e^{2 \sigma \varphi(x, t)}, \quad \forall(x, t) \in Q(0, \delta) .
\end{aligned}
$$

Here, $(U, V)$ is a vector-valued function and satisfies

$|U(x, t)|+|V(x, t)| \leq C\left(\sigma\left|\nabla_{x, t} u(x, t)\right|^{2}+\sigma^{3}|u(x, t)|^{2}\right) e^{2 \sigma \varphi(x, t)}, \quad \forall(x, t) \in Q(0, \delta)$.

Moreover, $V(x, 0)=0, \forall x \in \Omega$ if $u(x, 0)=0$ or $\partial_{t} u(x, 0)=0, \forall x \in \Omega$.

From this Lemma, we deduce the following Carleman estimate:

Corollary 2 Let $q \in C^{2}(\bar{\Omega})$ satisfy Condition 1 and $\varphi$ be defined by (4), with $\beta>0$ sufficiently small. Then, there exist $\beta_{0}>0$ and $\sigma_{0}>0$ such that, for all $\beta \leq \beta_{0}$, for all $\sigma \geq \sigma_{0}$, for all $\delta>0$ and for all $u \in H^{2}(Q(0, \delta))$ satisfying $u(x, 0)=0$ or $\partial_{t} u(x, 0)=0, \forall x \in \Omega$, we have

$\sigma\left\|u e^{\sigma \varphi}\right\|_{H^{1, \sigma}(Q(0, \delta))}^{2} \leq C\left(\left\|\left(\partial_{t}^{2} u-q \Delta u\right) e^{\sigma \varphi}\right\|_{L^{2}(Q(0, \delta))}^{2}+\sigma\left\|u e^{\sigma \varphi}\right\|_{H^{1, \sigma}(\partial Q(0, \delta) \backslash(\Omega \times\{0\}))}^{2}\right)$.

Proof We integrate $(16)$ over $Q(0, \delta)$ :

$\int_{Q(0, \delta)}\left(\sigma\left|\nabla_{x, t} u(x, t)\right|^{2}+\sigma^{3}|u(x, t)|^{2}\right) e^{2 \sigma \varphi(x, t)} d x d t$

$\leq-\int_{Q(0, \delta)}\left(\nabla \cdot U(x, t)-\partial_{t} V(x, t)+C\left|\partial_{t}^{2} u(x, t)-\mu(x) \delta u(x, t)\right|^{2} e^{2 \sigma \varphi(x, t)}\right) d x d t$.

We define now (see Figure 5) $\forall t \geq 0, \Omega_{t}(\varepsilon, \delta)=Q(\varepsilon, \delta) \cap(\Omega \times\{t\})$ and

$$
\forall x \in \Omega, \quad t_{x}^{\delta}=\left\{\begin{array}{cl}
\sqrt{\frac{\left|x-x_{0}\right|^{2}-\delta}{\beta}} & \text { if } \delta<\left|x-x_{0}\right|^{2}, \\
0 & \text { else. }
\end{array}\right.
$$

We notice that

$$
\begin{aligned}
\int_{Q(0, \delta)} \nabla \cdot U(x, t) d x d t & =\int_{0}^{\infty} \int_{\Omega_{t}(0, \delta)} \nabla \cdot U(x, t) d x d t=\int_{0}^{\infty} \int_{\partial \Omega_{t}(0, \delta)} U(x, t) \cdot n d x d t \\
& =\int_{\partial Q(0, \delta) \backslash(\Omega \times\{0\})} U(x, t) \cdot n d x d t
\end{aligned}
$$

and that

$$
\begin{aligned}
\int_{Q(0, \delta)} \partial_{t} V(x, t) d x d t & =\int_{\Omega} \int_{0}^{t_{x}^{\delta}} \partial_{t} V(x, t) d t d x=\int_{\Omega}\left(V\left(x, t_{x}^{\delta}\right)-V(x, 0)\right) d x \\
& =\int_{\partial Q(0, \delta) \backslash(\Omega \times\{0\})} V(x, t) d x d t .
\end{aligned}
$$



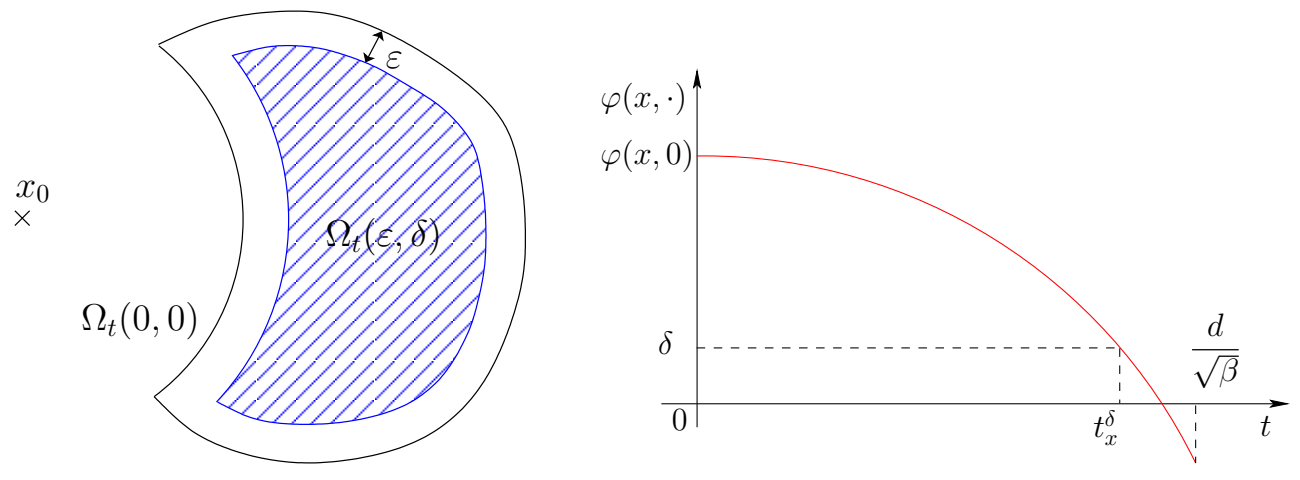

Figure 5. Definition of the domain $\Omega_{t}(\varepsilon, \delta)$ and the time $t_{x}^{\delta}$.

Thus,

$$
\begin{aligned}
-\int_{Q(0, \delta)} \nabla \cdot U(x, & t) d x d t-\int_{Q(0, \delta)} \partial_{t} V(x, t) d x d t \\
& \leq \int_{\partial Q(0, \delta) \backslash(\Omega \times\{0\})}(|U(x, t)|+|V(x, t)|) d x d t \\
& \leq C \int_{\partial Q(0, \delta) \backslash(\Omega \times\{0\})}\left(\sigma\left|\nabla_{x, t} u(x, t)\right|^{2}+\sigma^{3}|u(x, t)|^{2}\right) e^{2 \sigma \varphi(x, t)} d x d t .
\end{aligned}
$$

This achieves to prove the expected result.

For $\varepsilon>0$ and $\delta>0$, we introduce then the cut-off function $\chi_{2} \in C^{\infty}\left(\mathbb{R}^{3} \times \mathbb{R}\right)$ which satisfies $0 \leq \chi_{2} \leq 1$ and such that

$$
\chi_{2}(x, t)=\left\{\begin{array}{lll}
1, & \text { if } \quad(x, t) \in Q(2 \varepsilon, 2 \delta), \\
0, & \text { if } \quad(x, t) \in Q \backslash \overline{Q(\varepsilon, \delta)} .
\end{array}\right.
$$
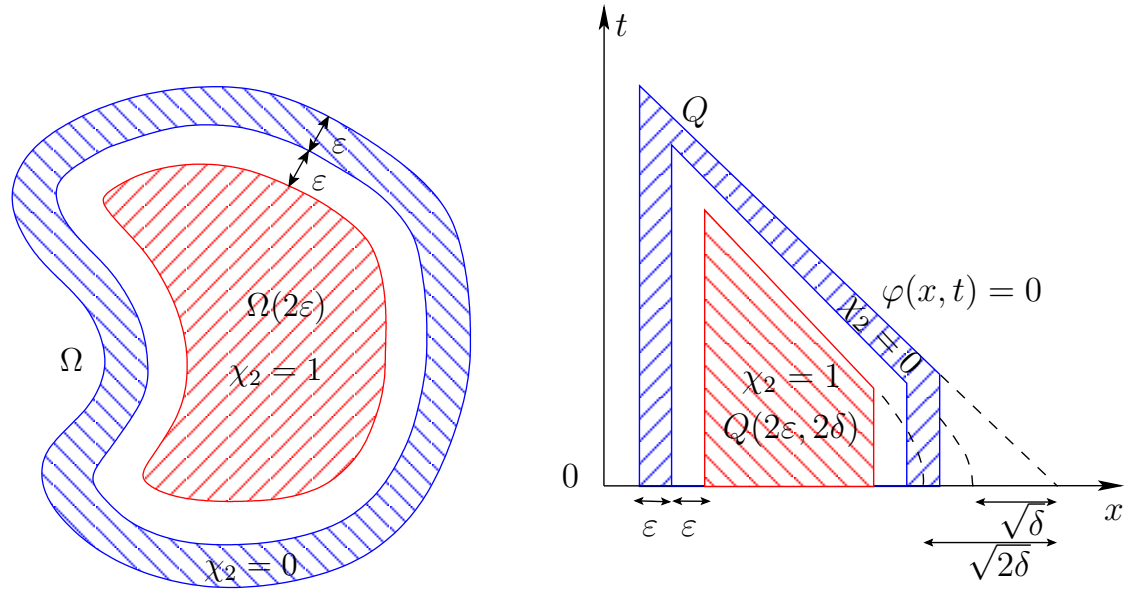

Figure 6. Definition of the cut-off function $\chi_{2}$. 
Figure 8 illustrates this cut-off function. Then, we set

$$
u_{i}^{*}(x, t)=\chi_{2}(x, t) \tilde{u}_{i}(x, t), \quad \forall i \in\{1,2,3\}, \quad \forall(x, t) \in \Omega \times(0,+\infty) .
$$

Thus, $u_{i}^{*}$ satisfies the equation

$$
\begin{aligned}
\partial_{t}^{2} u_{i}^{*}(x, t)-q_{i}(x) \Delta u_{i}^{*}(x, t) & \\
= & \chi(x, t)\left(\partial_{t}^{2} \tilde{u}_{i}(x, t)-\mu(x) \Delta \tilde{u}_{i}(x, t)\right)+2 \partial_{t} \chi(x, t) \partial_{t} \tilde{u}_{i}(x, t) \\
& +\tilde{u}_{i}(x, t)\left(\partial_{t}^{2} \chi(x, t)-\mu(x) \Delta \chi(x, t)\right)-2 \mu(x) \nabla \chi(x, t) \cdot \nabla \tilde{u}_{i}(x, t) \\
= & \chi(x, t)\left(q_{i}(x)\left(F_{i}(x, t)+A_{i}\left(u_{1}, u_{2}, u_{3}\right)(x, t)\right)+L_{i}\left(u_{i}\right)(x, t)\right)+\tilde{L}_{i}\left(\tilde{u}_{i}\right)(x, t),
\end{aligned}
$$

where $\tilde{L}_{i}$ is a first order integro-differential operator with coefficients bounded in $\Omega \times(0,+\infty)$. Since we supposed $u(x, 0)=0$ or $\partial_{t} u(x, 0)=0, \forall x \in \Omega$, and that, according to $(H 2)$, the coefficient $q_{i}$ satisfies Condition 1 , we can apply the Corollary 2 to $u_{i}^{*}$ in $Q(0, \delta / 2)$, i.e. there exists $\beta>0$ sufficiently small such that, for $\sigma>0$ sufficiently large, we have

$$
\sigma\left\|u_{i}^{*} e^{\sigma \varphi}\right\|_{H^{1, \sigma}(Q(\varepsilon, \delta))}^{2} \leq C\left\|\left(\partial_{t}^{2} u_{i}^{*}-q_{i} \Delta u_{i}^{*}\right) e^{\sigma \varphi}\right\|_{L^{2}(Q(\varepsilon, \delta))}^{2},
$$

without boundary term since $u_{i}^{*}$ is null in $Q \backslash \overline{Q(\varepsilon, \delta)}$.

\subsection{Return to the initial variable $u$}

3.4.1. Upper bound for the right hand side We take into account the fact that

- all the coefficients $\lambda, \mu, \tilde{\lambda}, \tilde{\mu}$ and $\chi$ are bounded in $\Omega \times(0,+\infty)$ according to $(H 1)$,

- all the operators $A_{i}, L_{i}$ and $\tilde{L}_{i}$ are of order inferior or equal to 1 ,

- Lemma 1 holds,

to calculate:

$$
\begin{aligned}
\left\|\left(\partial_{t}^{2} u_{i}^{*}-q_{i} \Delta u_{i}^{*}\right) e^{\sigma \varphi}\right\|_{L^{2}(Q(\varepsilon, \delta))}^{2} & \\
& =\left\|\left(\chi\left(\mu\left(F_{i}+A_{i}\left(u_{1}, u_{2}, u_{3}\right)\right)+L_{i}\left(u_{i}\right)\right)+\tilde{L}_{i}\left(\tilde{u}_{i}\right)\right) e^{\sigma \varphi}\right\|_{L^{2}(Q(\varepsilon, \delta))}^{2} .
\end{aligned}
$$

Then,

$\sigma\left\|u_{i}^{*} e^{\sigma \varphi}\right\|_{H^{1, \sigma}(Q(\varepsilon, \delta))}^{2} \leq C\left\|F_{i} e^{\sigma \varphi}\right\|_{L^{2}(Q)}^{2}+C \sum_{1 \leq j \leq 3}\left\|u_{j} e^{\sigma \varphi}\right\|_{H^{1, \sigma}(Q(\varepsilon, \delta))}^{2}$.

3.4.2. Lower bound for the left hand side We use again the change of variable (15), for all $(x, t) \in \Omega \times(0,+\infty)$ :

$$
u_{i}(x, t)=\frac{1}{q_{i}(x)} \tilde{u}_{i}(x, t)+\int_{0}^{t} \frac{\tilde{q}_{i}(x, t-s)}{q_{i}(x)} u_{i}(x, s) d s, \quad \forall i \in\{1,2,3\},
$$

Then, we can write, taking into account (H2) and thanks to Lemma $1, \forall i \in\{1,2,3\}$ :

$$
\begin{aligned}
\int_{Q(\varepsilon, \delta)}\left|u_{i}(x, t)\right|^{2} e^{2 \sigma \varphi(x, t)} d x d t & \leq C \int_{Q(\varepsilon, \delta)}\left|\tilde{u}_{i}(x, t)\right|^{2} e^{2 \sigma \varphi(x, t)} d x d t \\
& +\frac{C}{\sigma} \int_{Q(\varepsilon, \delta)}\left|u_{i}(x, t)\right|^{2} e^{2 \sigma \varphi(x, t)} d x d t .
\end{aligned}
$$

For $\sigma$ sufficiently large, we obtain

$$
\int_{Q(\varepsilon, \delta)}\left|u_{i}(x, t)\right|^{2} e^{2 \sigma \varphi(x, t)} d x d t \leq C \int_{Q(\varepsilon, \delta)}\left|\tilde{u}_{i}(x, t)\right|^{2} e^{2 \sigma \varphi(x, t)} d x d t .
$$


In the same way,

$$
\begin{aligned}
& \int_{Q(\varepsilon, \delta)}\left|\nabla_{x, t} u_{i}(x, t)\right|^{2} e^{2 \sigma \varphi(x, t)} d x d t \\
& \leq C \int_{Q(\varepsilon, \delta)}\left(\left|\tilde{u}_{i}(x, t)\right|^{2}+\left|\nabla_{x, t} \tilde{u}_{i}(x, t)\right|^{2}\right) e^{2 \sigma \varphi(x, t)} d x d t
\end{aligned}
$$

which allows to write, $\forall i \in\{1,2,3\}$ :

$$
\left\|u_{i} e^{\sigma \varphi}\right\|_{H^{1, \sigma}(Q(\varepsilon, \delta))}^{2} \leq C\left\|\tilde{u}_{i} e^{\sigma \varphi}\right\|_{H^{1, \sigma}(Q(\varepsilon, \delta))}^{2} .
$$

In addition, $\chi_{2}=1 \operatorname{sur} Q(2 \varepsilon, 2 \delta)$, so we have $\tilde{u}_{i}=u_{i}^{*}$. Hence $\forall i \in\{1,2,3\}$,

$\left\|\tilde{u}_{i} e^{\sigma \varphi}\right\|_{H^{1, \sigma}(Q(\varepsilon, \delta))}^{2}=\left\|u_{i}^{*} e^{\sigma \varphi}\right\|_{H^{1, \sigma}(Q(2 \varepsilon, 2 \delta))}^{2}+\left\|\tilde{u}_{i} e^{\sigma \varphi}\right\|_{H^{1, \sigma}(Q(\varepsilon, \delta) \backslash Q(2 \varepsilon, 2 \delta))}^{2}$.

Finally, using (18), (19) and (20) and again Lemma 1, we obtain

$\sum_{1 \leq i \leq 3} \sigma\left\|u_{i} e^{\sigma \varphi}\right\|_{H^{1, \sigma}(Q(\varepsilon, \delta))}^{2}$

$\leq C \sum_{1 \leq i \leq 3}\left(\left\|F_{i} e^{\sigma \varphi}\right\|_{L^{2}(Q)}^{2}+\left\|u_{i} \cdot e^{\sigma \varphi}\right\|_{H^{1, \sigma}(Q(\varepsilon, \delta))}^{2}+\sigma\left\|u_{i} e^{\sigma \varphi}\right\|_{H^{1, \sigma}(Q(\varepsilon, \delta) \backslash Q(2 \varepsilon, 2 \delta))}^{2}\right)$,

and thanks to the Carleman weights, we absorb the second term of the right hand side in the left hand side. Finally, we need the following lemma:

Lemma 5 Let $\varphi$ defined by (4). There exist $C>0$ and $\sigma_{0}>0$ such that, for all $\sigma \geq \sigma_{0}$ and for all $u \in H^{2}(Q)$, we have

$$
\begin{aligned}
& \frac{1}{\sigma}\left\|u e^{\sigma \varphi}\right\|_{H_{x}^{2, \sigma}(Q(2 \varepsilon, 2 \delta)}^{2} \\
& \quad \leq C\left(\sigma\left\|u e^{\sigma \varphi}\right\|_{H_{x}^{1, \sigma}(Q(\varepsilon, \delta))}^{2}+\left\|\nabla(\nabla \cdot u) e^{\sigma \varphi}\right\|_{L^{2}(Q(\varepsilon, \delta))}^{2}+\left\|\nabla(\nabla \wedge u) e^{\sigma \varphi}\right\|_{L^{2}(Q(\varepsilon, \delta))}^{2}\right) .
\end{aligned}
$$

Proof With $\chi_{2}$ defined in (17), we compute, for all $(x, t) \in Q$,

$$
\begin{aligned}
& \Delta\left(\chi_{2}(x, t) u(x, t) e^{\sigma \varphi(x, t)}\right)=2\left(\sigma \chi_{2}(x, t) \nabla \varphi(x, t)+\nabla \chi_{2}(x, t)\right) \cdot \nabla u(x, t) e^{\sigma \varphi(x, t)} \\
& \quad+\left(\sigma^{2} \chi_{2}(x, t)|\nabla \varphi(x, t)|^{2}+\sigma \chi_{2}(x, t) \Delta \varphi(x, t)+\Delta \chi_{2}(x, t)\right) u(x, t) e^{\sigma \varphi(x, t)} \\
& +\left(\sigma \nabla \varphi(x, t) \cdot \nabla \chi_{2}(x, t)\right) u(x, t) e^{\sigma \varphi(x, t)}+\chi_{2}(x, t) \Delta u(x, t) e^{\sigma \varphi(x, t)} .
\end{aligned}
$$

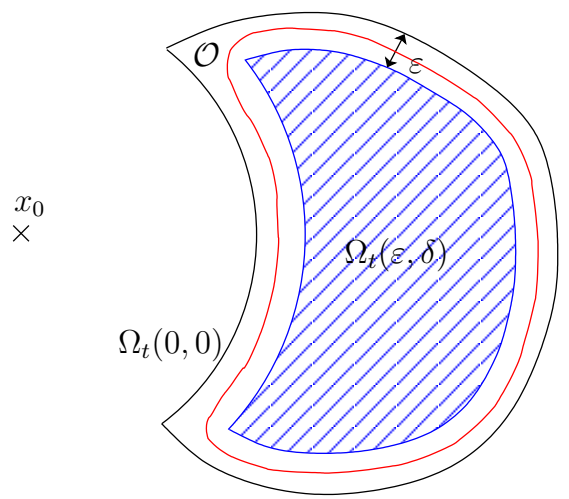

Figure 7. Definition of the open $\mathcal{O}$. 
For all $t \geq 0$, we can find an open $\mathcal{O}$ with smooth boundary (at least $C^{1,1}$ ) such that $\Omega_{t}(\varepsilon, \delta) \subset \mathcal{O} \subset \Omega_{t}(0,0)$. Since, for $t$ fixed, the function $\chi_{2}(\cdot, t) u(\cdot, t) e^{\sigma \varphi(\cdot, t)}$ is in $H^{2}(\mathcal{O}) \cap H_{0}^{1}(\mathcal{O})$, we can write:

$$
\left\|\chi_{2}(\cdot, t) u(\cdot, t) e^{\sigma \varphi(\cdot, t)}\right\|_{H^{2}(\mathcal{O})} \leq C\left\|\Delta\left(\chi_{2}(\cdot, t) u(\cdot, t) e^{\sigma \varphi(\cdot, t)}\right)\right\|_{L^{2}(\mathcal{O})}
$$

If we integrate inequality (21) for $t \in(0,+\infty)$ and notice that $\chi_{1}=0$ in $\mathcal{O} \backslash \Omega_{t}(\varepsilon, \delta)$, we obtain

$$
\begin{aligned}
& \left\|\chi_{2} u e^{\sigma \varphi}\right\|_{H_{x}^{2, \sigma}(Q(\varepsilon, \delta))}^{2} \\
& \quad \leq\left\|u e^{\sigma \varphi}\right\|_{L^{2}\left(0,+\infty ; H^{2}\left(\Omega_{t}(\varepsilon, \delta)\right)\right)}^{2}+\sigma^{2}\left\|\nabla\left(u e^{\sigma \varphi}\right)\right\|_{L^{2}(Q(\varepsilon, \delta))}^{2}+\sigma^{4}\left\|u e^{\sigma \varphi}\right\|_{L^{2}(Q(\varepsilon, \delta))}^{2} \\
& \quad \leq C\left(\left\|e^{\sigma \varphi} \Delta u\right\|_{L^{2}(Q(\varepsilon, \delta))}^{2}+\sigma^{2}\left\|e^{\sigma \varphi} \nabla u\right\|_{L^{2}(Q(\varepsilon, \delta))}^{2}+\sigma^{4}\left\|u e^{\sigma \varphi}\right\|_{L^{2}(Q(\varepsilon, \delta))}^{2}\right) .
\end{aligned}
$$

We use the classical formula

$$
\Delta u=\nabla(\nabla \cdot u)-\nabla \wedge(\nabla \wedge u),
$$

and the fact that $\chi_{2}=1$ in $Q(2 \varepsilon, 2 \delta)$ to write

$$
\begin{aligned}
& \left\|u e^{\sigma \varphi}\right\|_{H_{x}^{2, \sigma}(Q(2 \varepsilon, 2 \delta))}^{2} \leq\left\|\chi_{2} u e^{\sigma \varphi}\right\|_{H_{x}^{2, \sigma}(Q(\varepsilon, \delta))}^{2} \\
& \quad \leq C\left(\left\|e^{\sigma \varphi} \nabla(\nabla \cdot u)\right\|_{L^{2}(Q(\varepsilon, \delta))}^{2}+\left\|e^{\sigma \varphi} \nabla(\nabla \wedge u)\right\|_{L^{2}(Q(\varepsilon, \delta))}^{2}+\sigma^{2}\left\|u e^{\sigma \varphi}\right\|_{H_{x}^{1, \sigma}(Q(\varepsilon, \delta))}^{2}\right) .
\end{aligned}
$$

This is the expected result.

Using this lemma, we can finally write:

$$
\begin{aligned}
& \frac{1}{\sigma}\left\|u e^{\sigma \varphi}\right\|_{H_{x}^{2, \sigma}(Q(2 \varepsilon, 2 \delta))}^{2} \\
& \leq C\left(\sigma\left\|u_{1} e^{\sigma \varphi}\right\|_{H_{x}^{1, \sigma}(Q(\varepsilon, \delta))}^{2}+\left\|\nabla u_{2} e^{\sigma \varphi}\right\|_{L^{2}(Q(\varepsilon, \delta))}^{2}+\left\|\nabla u_{3} e^{\sigma \varphi}\right\|_{L^{2}(Q(\varepsilon, \delta))}^{2}\right) \\
& \leq C \sum_{1 \leq i \leq 3} \sigma\left\|u_{i} e^{\sigma \varphi}\right\|_{H^{1, \sigma}(Q(\varepsilon, \delta))}^{2} \\
& \leq C \sum_{1 \leq i \leq 3}\left(\left\|F_{i} e^{\sigma \varphi}\right\|_{L^{2}(Q)}^{2}+\sigma\left\|u_{i} e^{\sigma \varphi}\right\|_{H^{1, \sigma}(Q(\varepsilon, \delta) \backslash Q(2 \varepsilon, 2 \delta))}^{2}\right), \\
& \frac{1}{\sigma}\left\|u e^{\sigma \varphi}\right\|_{H_{x}^{2, \sigma}(Q(\varepsilon, \delta))}^{2}=\frac{1}{\sigma}\left\|u e^{\sigma \varphi}\right\|_{H_{x}^{2, \sigma}(Q(2 \varepsilon, 2 \delta))}^{2}+\frac{1}{\sigma}\left\|u e^{\sigma \varphi}\right\|_{H_{x}^{2, \sigma}(Q(\varepsilon, \delta) \backslash Q(2 \varepsilon, 2 \delta))}^{2} \\
& \leq C \sum_{1 \leq i \leq 3}\left(\left\|F_{i} e^{\sigma \varphi}\right\|_{L^{2}(Q)}^{2}+\sigma\left\|u_{i} e^{\sigma \varphi}\right\|_{H^{1, \sigma}(Q(\varepsilon, \delta) \backslash Q(2 \varepsilon, 2 \delta))}^{2}\right) .
\end{aligned}
$$

Moreover, we have

$$
\begin{aligned}
& \sum_{1 \leq i \leq 3} \sigma\left\|u_{i} e^{\sigma \varphi}\right\|_{H^{1, \sigma}(Q(\varepsilon, \delta) \backslash Q(2 \varepsilon, 2 \delta))}^{2} \\
& \quad=\sum_{1 \leq i \leq 3}\left(\sigma\left\|u_{i} e^{\sigma \varphi}\right\|_{H^{1, \sigma}(Q(\varepsilon, \delta) \backslash Q(2 \varepsilon, \delta)}^{2}+\sigma\left\|u_{i} e^{\sigma \varphi}\right\|_{H^{1, \sigma}(Q(\varepsilon, \delta) \backslash Q(\varepsilon, 2 \delta))}^{2}\right) \\
& \quad \leq e^{C \sigma}\|u\|_{H^{2}(Q(\varepsilon, \delta) \backslash Q(2 \varepsilon, \delta))}^{2}+\sigma^{3} e^{2 \sigma \delta}\|u\|_{H^{2}(Q)}^{2}, \\
& \text { since }
\end{aligned}
$$

$$
\varphi(x, t) \leq \delta, \quad \forall(x, t) \in Q(\varepsilon, \delta) \backslash Q(\varepsilon, 2 \delta) .
$$

Thus

$$
\begin{aligned}
& \frac{1}{\sigma}\left\|u e^{\sigma \varphi}\right\|_{H_{x}^{2, \sigma}(Q(\varepsilon, \delta))}^{2} \\
& \leq C\left(\left\|F e^{\sigma \varphi}\right\|_{L^{2}(Q)}^{2}+\left\|(\nabla F) e^{\sigma \varphi}\right\|_{L^{2}(Q)}^{2}+e^{C \sigma}\|u\|_{H^{2}(Q(\varepsilon, \delta) \backslash Q(2 \varepsilon, \delta))}^{2}+\sigma^{3} e^{2 \sigma \delta}\|u\|_{H^{2}(Q)}^{2}\right),
\end{aligned}
$$
and this concludes the proof of Theorem 2. 


\section{Proof of Theorem 3}

In this section, we prove the unique continuation result of Theorem 3. It consists in transforming the integro-differential hyperbolic system (1)-(2) into an elliptic one thanks to a FBI type transform. Then, we show a Carleman estimate (Theorem 4) for the resulting integro-differential elliptic operator, using the same techniques as in the proof of Theorem 2. And we use this Carleman estimate to obtain interpolation inequalities which link the value of the solution in an interior domain near the boundary to the one on the boundary. Finally, we come back to the solution of the initial problem by a series of inequalities.

Let $u$ be the solution of

$$
\mathcal{P} u(x, t)=R(x, t), \quad \forall(x, t) \in \Omega \times(0,+\infty),
$$

with null initial and boundary conditions and let assume that $(H 1)$ and $(H 6)$ are satisfied.

\subsection{Transform the hyperbolic system into an elliptic one}

Let us choose $\varepsilon>0$ such that $\Omega \backslash \Omega(3 \varepsilon) \subset \omega$.
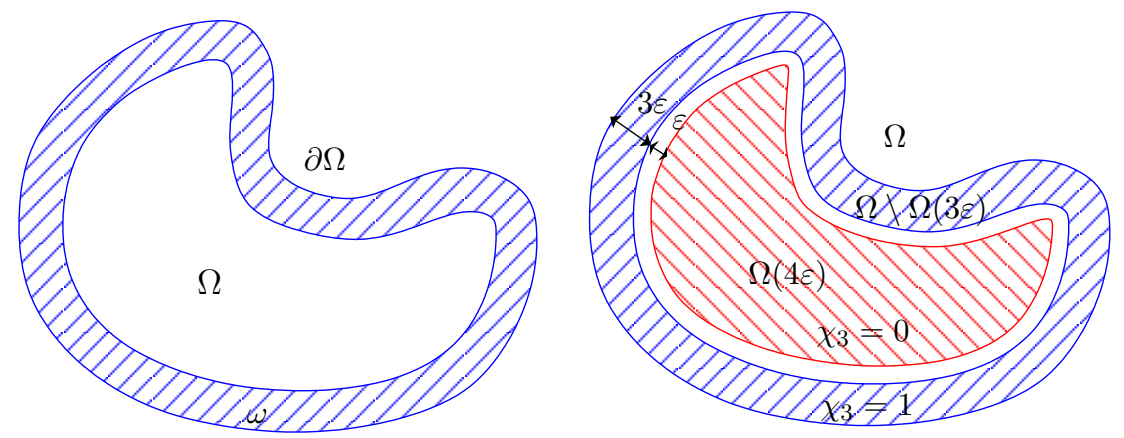

Figure 8. Definition of the neighborhood $\omega$ of $\partial \Omega$ and the cut-off function $\chi_{3}$.

We introduce the cut-off function $\chi_{3} \in C_{0}^{\infty}\left(\mathbb{R}^{3}\right)$ (see Figure 8) which satisfies $0 \leq \chi_{3} \leq 1$ and is such that

$$
\chi_{3}(x)=\left\{\begin{array}{lll}
0, & \text { if } & x \in \Omega(4 \varepsilon), \\
1, & \text { if } & x \in \Omega \backslash \overline{\Omega(3 \varepsilon)},
\end{array}\right.
$$

and we set

$$
u^{*}(x, t)=\chi_{3}(x) u(x, t), \quad \forall(x, t) \in \Omega \times(0,+\infty) .
$$

Thus, the new variable $u^{*}$ satisfies the following equation:

$$
\begin{gathered}
\mathcal{P} u^{*}(x, t)=\chi_{3}(x) \mathcal{P} u(x, t)+\left[\mathcal{P}, \chi_{3}\right] u(x, t) \\
\Longrightarrow \partial_{t}^{2} u^{*}(x, t)-\mathcal{L}(x) u^{*}(x, t)+\int_{0}^{t} \tilde{\mathcal{L}}(x, s) u^{*}(x, t-s) d s=\left[\mathcal{P}(x, t), \chi_{3}\right] u(x, t),
\end{gathered}
$$

since, according to $(H 6), R(x, t)$ is equal to zero in $\omega$. We have introduced the following operators:

$$
\mathcal{L}(x) \star=\nabla \cdot\left(\mu(x)\left(\nabla \star+\nabla \star^{T}\right)+\lambda(x)(\nabla \cdot \star) I\right),
$$


Logarithmic stability in determination of a $3 D$ viscoelastic coefficient

$$
\tilde{\mathcal{L}}(x, t) \star=\nabla \cdot\left(p(x) h(t)\left(\nabla \star+\nabla \star^{T}\right)+\tilde{\lambda}(x, t)(\nabla \cdot \star) I\right) .
$$

We define a special transformation which is inspired from the classical Fourier-BrosIagolnitzer transform [25]:

Definition 3 Let $T>1$ and $0<\eta<\frac{1}{6}$. We introduce $\widetilde{Q}=\Omega \times\left(-\frac{T}{2}, \frac{5 T}{2}\right) \times(-3 \eta, 3 \eta)$. Then, $\forall(x, t, r) \in \widetilde{Q}$, we define

$$
\left(\mathcal{F}_{\gamma} u^{*}\right)(x, t, r)=\sqrt{\frac{\gamma}{2 \pi}} \int_{0}^{+\infty} e^{-\frac{\gamma}{2}(t+i r-y)^{2}} \theta(t-y) u^{*}(x, y) d y,
$$

where $\theta \in C^{\infty}(\mathbb{R}), 0 \leq \theta \leq 1$,

$$
\theta(z)= \begin{cases}1, & \text { if } \quad z>-\frac{T}{2}+3 \eta \\ 0, & \text { if } \quad z<-\frac{T}{2}+\frac{3 \eta}{2} .\end{cases}
$$

This function is plotted in Figure 9.

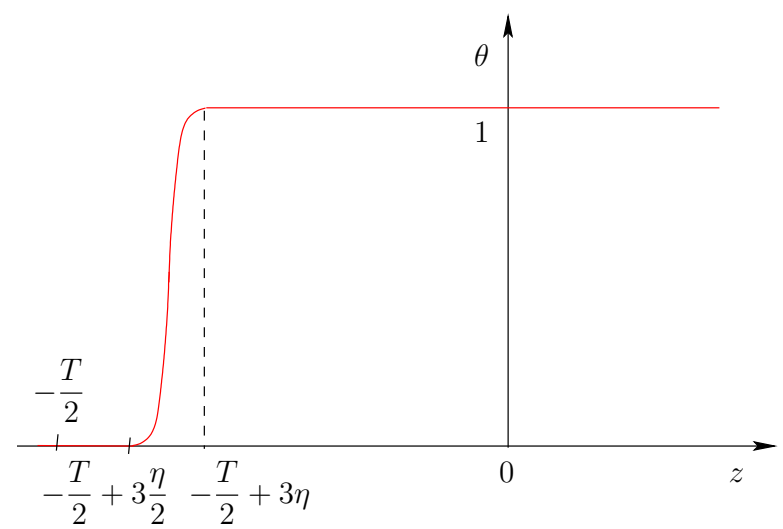

Figure 9. Definition of the cut-off function $\theta$.

Thanks to this transform, we will convert locally the hyperbolic system (23) into an elliptic system. Firstly, we notice that, $\forall(x, t, r) \in \widetilde{Q}$,

$$
\begin{aligned}
\partial_{r}\left(\mathcal{F}_{\gamma} u^{*}\right)(x, t, r) & =\sqrt{\frac{\gamma}{2 \pi}} \int_{0}^{+\infty}-i \gamma(t+i r-y) e^{-\frac{\gamma}{2}(t+i r-y)^{2}} \theta(t-y) u^{*}(x, y) d y \\
& =\sqrt{\frac{\gamma}{2 \pi}} \int_{0}^{+\infty}-i \partial_{y}\left(e^{-\frac{\gamma}{2}(t+i r-y)^{2}}\right) \theta(t-y) u^{*}(x, y) d y \\
& =\sqrt{\frac{\gamma}{2 \pi}} \int_{0}^{+\infty} i e^{-\frac{\gamma}{2}(t+i r-y)^{2}} \partial_{y}\left(\theta(t-y) u^{*}(x, y)\right) d y
\end{aligned}
$$

The boundary terms of the integration by parts vanish because $u^{*}(x, 0)=0$ and $e^{-\frac{\gamma}{2}(t+i r-y)^{2}} \longrightarrow 0$ if $y \longrightarrow \infty$. Moreover $\theta(t-y)=0$ if $y \geq T$. Then,

$$
\begin{aligned}
\partial_{r}^{2}\left(\mathcal{F}_{\gamma} u^{*}\right)(x, t, r) & =-\sqrt{\frac{\gamma}{2 \pi}} \int_{0}^{+\infty} e^{-\frac{\gamma}{2}(t+i r-y)^{2}} \partial_{y}^{2}\left(\theta(t-y) u^{*}(x, y)\right) d y \\
& =-\left(\mathcal{F}_{\gamma}\left(\partial_{y}^{2} u^{*}\right)\right)(x, t, r)-F_{\gamma}(x, t, r), \quad \forall(x, t, r) \in \widetilde{Q},
\end{aligned}
$$


where

$F_{\gamma}(x, t, r)=\sqrt{\frac{\gamma}{2 \pi}} \int_{0}^{+\infty} e^{-\frac{\gamma}{2}(t+i r-y)^{2}}\left(\theta^{\prime \prime}(t-y) u^{*}(x, y)-2 \theta^{\prime}(t-y) \partial_{y} u^{*}(x, y)\right) d y$.

Similarly, we can write:

$$
\partial_{t}^{2}\left(\mathcal{F}_{\gamma} u^{*}\right)(x, t, r)=\left(\mathcal{F}_{\gamma}\left(\partial_{y}^{2} u^{*}\right)\right)(x, t, r), \quad \forall(x, t, r) \in \widetilde{Q},
$$

and it is easy to see that

$$
\mathcal{L}(x)\left(\mathcal{F}_{\gamma} u^{*}\right)(x, t, r)=\left(\mathcal{F}_{\gamma}\left(\mathcal{L}(x) u^{*}\right)\right)(x, t, r), \quad \forall(x, t, r) \in \widetilde{Q} .
$$

We have now to look at the integral term

$$
\begin{aligned}
\mathcal{F}_{\gamma}( & \left.\int_{0}^{y} \tilde{\mathcal{L}}(x, s) u^{*}(x, y-s) d s\right)(x, t, r) \\
& =\sqrt{\frac{\gamma}{2 \pi}} \int_{0}^{+\infty} e^{-\frac{\gamma}{2}(t+i r-y)^{2}} \theta(t-y) \int_{0}^{y} \tilde{\mathcal{L}}(x, s) u^{*}(x, y-s) d s d y \\
& =\sqrt{\frac{\gamma}{2 \pi}} \int_{0}^{+\infty} \tilde{\mathcal{L}}(x, s) \int_{s}^{+\infty} e^{-\frac{\gamma}{2}(t+i r-y)^{2}} \theta(t-y) u^{*}(x, y-s) d y d s \\
& =\int_{0}^{+\infty} \tilde{\mathcal{L}}(x, s) \sqrt{\frac{\gamma}{2 \pi}} \int_{0}^{+\infty} e^{-\frac{\gamma}{2}(t-s+i r-z)^{2}} \theta(t-s-z) u^{*}(x, z) d z d s \\
& =\int_{0}^{+\infty} \tilde{\mathcal{L}}(x, s)\left(\mathcal{F}_{\gamma} u^{*}\right)(x, t-s, r) d s=\int_{-T / 2}^{t} \tilde{\mathcal{L}}(x, t-s)\left(\mathcal{F}_{\gamma} u^{*}\right)(x, s, r) d s .
\end{aligned}
$$

The main advantage of this transformation with respect to the FBI transform is that it transforms the convolution product of two functions into the convolution of the first function by the transform of the second function. The function $u_{\gamma}=\mathcal{F}_{\gamma} u^{*}$, function of three variables $(x, t, r)$, satisfies then a system of elliptic integro-differential equations, $\forall(x, t, r) \in \widetilde{Q}:$

$$
\begin{aligned}
\mathcal{Q} u_{\gamma}(x, t, r)= & -2 \partial_{r}^{2} u_{\gamma}(x, t, r)-\partial_{t}^{2} u_{\gamma}(x, t, r)-\mathcal{L}(x) u_{\gamma}(x, t, r) \\
& +\int_{-T / 2}^{t} \tilde{\mathcal{L}}(x, t-s) u_{\gamma}(x, s, r) d s=2 F_{\gamma}(x, t, s)+G_{\gamma}(x, t, s),
\end{aligned}
$$

where

$G_{\gamma}(x, t, r)=\sqrt{\frac{\gamma}{2 \pi}} \int_{0}^{+\infty} e^{-\frac{\gamma}{2}(t+i r-y)^{2}} \theta(t-y)\left[\mathcal{P}(x, t), \chi_{3}\right] \hat{u}(x, y) d y$,

with the boundary conditions:

$$
\left\{\begin{array}{l}
u_{\gamma}(x, t, r)=0, \quad \forall(x, t, r) \in \partial \Omega \times\left(-\frac{T}{2}, \frac{5 T}{2}\right) \times(-3 \eta, 3 \eta), \\
u_{\gamma}(x, t, r)=0, \quad \forall(x, t, r) \in \Omega \times\left\{-\frac{T}{2}\right\} \times(-3 \eta, 3 \eta) .
\end{array}\right.
$$

We notice then that

$$
G_{\gamma}(x, t, r)=0, \quad \forall x \in \Omega \backslash \overline{\Omega(3 \varepsilon)},
$$

because $\left[\mathcal{P}(x, t), \chi_{3}\right]$ only involves the derivatives of $\chi_{3}$ and its support is in $\overline{\Omega(3 \varepsilon)} \backslash$ $\Omega(4 \varepsilon)$. We also have

$$
\left\|F_{\gamma}\right\|_{H^{1}(\widetilde{Q})} \leq C e^{-m \gamma T}\left\|u^{*}\right\|_{H^{2}(\Omega \times(0,3 T))}
$$


as well as

$$
\left\|u_{\gamma}\right\|_{H^{2}(\widetilde{Q})} \leq C e^{C \gamma}\left\|u^{*}\right\|_{H^{2}(\Omega \times(0,3 T))},
$$

where $C$ depends of $\eta, T, \Omega$ but not of $\gamma$ and where $m$ is independent of $T$. Indeed,

$\left\|F_{\gamma}\right\|_{L^{2}(\widetilde{Q})}^{2}=$

$\frac{\gamma}{2 \pi} \int_{\widetilde{Q}}\left|\int_{0}^{+\infty} e^{-\frac{\gamma}{2}(t+i r-y)^{2}}\left(\theta^{\prime \prime}(t-y) u^{*}(x, y)-2 \theta^{\prime}(t-y) \partial_{y} u^{*}(x, y)\right) d y\right|^{2} d r d t d x$.

with

$$
\begin{aligned}
\mid \int_{0}^{+\infty} & \left.e^{-\frac{\gamma}{2}(t+i r-y)^{2}}\left(\theta^{\prime \prime}(t-y) u^{*}(x, y)-2 \theta^{\prime}(t-y) \partial_{y} u^{*}(x, y)\right) d y\right|^{2} \\
\leq & \left(\int_{y \geq 0,-T / 2+3 \eta / 2 \leq t-y \leq-T / 2+3 \eta}\left|e^{-\frac{\gamma}{2}(t+i r-y)^{2}}\right|^{2} d y\right) \\
& \times\left(\int_{0}^{t+T / 2}\left|\theta^{\prime \prime}(t-y) u^{*}(x, y)-2 \theta^{\prime}(t-y) \partial_{y} u^{*}(x, y)\right|^{2} d y\right) \\
\leq & \left(\int_{y \geq 0,-T / 2+3 \eta / 2 \leq t-y \leq-T / 2+3 \eta} e^{-\gamma\left((t-y)^{2}-r^{2}\right)} d y\right) C\left\|u^{*}(x, \cdot)\right\|_{H^{1}(0,3 T)}^{2} \\
\leq & C e^{-m \gamma T}\left\|u^{*}(x, \cdot)\right\|_{H^{1}(0,3 T)}^{2}, \quad \forall(x, t, r) \in \widetilde{Q} .
\end{aligned}
$$

Indeed, $(t-y)^{2} \geq\left(-\frac{T}{2}+3 \eta\right)^{2}$ and $r^{2} \leq 9 \eta^{2}$ therefore $(t-y)^{2}-r^{2} \geq \frac{T}{4}(T-6 \eta)$. Thus, if we suppose $T>1$, since $\eta<1 / 6$, then there exists $m>0$ independent of $T$ such that $(t-y)^{2}-r^{2} \geq m T$. We can do the same work for the derivatives of $F_{\gamma}$. Likewise, we have

$\left\|u_{\gamma}\right\|_{L^{2}(\widetilde{Q})}^{2}=\int_{\Omega} \int_{-T / 2}^{5 T / 2} \int_{-3 \eta}^{3 \eta}\left|\sqrt{\frac{\gamma}{2 \pi}} \int_{0}^{+\infty} e^{-\frac{\gamma}{2}(t+i r-y)^{2}} \theta(t-y) u^{*}(x, y) d y\right|^{2} d r d t d x$

with, $\forall(x, t, r) \in \widetilde{Q}$,

$$
\begin{aligned}
& \left|\int_{0}^{+\infty} \quad e^{-\frac{\gamma}{2}(t+i r-y)^{2}} \theta(t-y) u^{*}(x, y) d y\right|^{2} \\
& \quad \leq\left(\int_{0}^{t+T / 2}\left|e^{-\frac{\gamma}{2}(t+i r-y)^{2}}\right|^{2} d y\right)\left(\int_{0}^{t+T / 2}\left|\theta(t-y) u^{*}(x, y)\right|^{2} d y\right) \\
& \quad \leq\left(\int_{0}^{3 T} e^{-\gamma\left((t-y)^{2}-r^{2}\right)} d y\right) C\left\|u^{*}(x, \cdot)\right\|_{L^{2}(0,3 T)}^{2} \leq C e^{C \gamma}\left\|u^{*}(x, \cdot)\right\|_{L^{2}(0,3 T)}^{2}
\end{aligned}
$$

And we use the same procedure for the derivatives of $u_{\gamma}$ to obtain the desired result.

\subsection{Prove a Carleman estimate}

We write a Carleman estimate for the system of elliptic integro-differential equations (24), applying the same methods we used in the hyperbolic case. That is, we combine the decoupling of the equations proposed by [14] with the change of variables of [10], and we use a classical Carleman estimate for a scalar elliptic equation (Lemma 2). The difference is that here we have 3 variables $(x, t, r)$, so the weight function has to be modified. Let us introduce first some notations: 
- Domains

For $T>0$ and $\eta>0$, we note

- Norms

$$
\begin{aligned}
& \widetilde{Q}=\Omega \times\left(-\frac{T}{2}, \frac{5 T}{2}\right) \times(-3 \eta, 3 \eta), \\
& \widetilde{\Sigma}=\partial \Omega \times\left(-\frac{T}{2}, \frac{5 T}{2}\right) \times(-3 \eta, 3 \eta) .
\end{aligned}
$$

$$
\begin{aligned}
& \|\star\|_{H^{k, \sigma}(\widetilde{Q})}^{2}=\sum_{|\alpha| \leq k} \sigma^{2(k-|\alpha|)}\left\|\partial^{\alpha} \star\right\|_{L^{2}(\widetilde{Q})}^{2}, \\
& \|\star\|_{H^{k, \sigma}(\widetilde{\Sigma})}^{2}=\sum_{|\alpha| \leq k} \sigma^{2(k-|\alpha|)}\left\|\partial^{\alpha} \star\right\|_{L^{2}(\widetilde{\Sigma})}^{2} .
\end{aligned}
$$

Definition 4 (a Carleman weight function) Let $x_{0} \in \mathbb{R}^{3} \backslash \bar{\Omega}$ and $\xi>0$. We introduce a function $\psi$ in the following way:

$$
\psi(x, t, r)=\left|x-x_{0}\right|^{2}+\left(t+\frac{T}{2}\right)^{2}+r^{2}, \quad \forall(x, t, r) \in \widetilde{Q},
$$

and we set

$$
\varphi(x, t, r)=e^{-\xi \psi(x, t, r)}, \quad \forall(x, t, r) \in \widetilde{Q} .
$$

Because we introduced this new weight function, we must now check that we still have a result, similar to to one of Lemma 1, to bound the integral terms in the proof of the Carleman estimate. It is given by the following lemma:

Lemma 6 Let $\varphi$ be defined by (28), $\sigma>0$ and $u \in L^{2}(\widetilde{Q})$. Then, there exists a constant $C>0$, independent of $\sigma$, such that

$\int_{\widetilde{Q}}\left(\int_{-T / 2}^{t}|u(x, s, r)| d s\right)^{2} e^{2 \sigma \varphi(x, t, r)} d x d t d r \leq \frac{C}{\sigma} \int_{\widetilde{Q}}|u(x, t, r)|^{2} e^{2 \sigma \varphi(x, t)} d x d t d r$.

Proof Using the Cauchy-Schwarz inequality, we can write:

$$
\begin{aligned}
& \int_{\widetilde{Q}}\left(\int_{-T / 2}^{t}|u(x, s, r)| d s\right)^{2} e^{2 \sigma \varphi(x, t, r)} d x d t d r \\
& \leq \int_{\widetilde{Q}}\left(\int_{-T / 2}^{t}|u(x, s, r)|^{2} d s\right)\left(t+\frac{T}{2}\right) e^{2 \sigma \varphi(x, t, r)} d x d t d r .
\end{aligned}
$$

We notice then that, $\forall(x, t, r) \in \widetilde{Q}$,

$\left(t+\frac{T}{2}\right) e^{2 \sigma \varphi(x, t, r)} \leq-\frac{e^{\xi \psi(x, t, r)}}{4 \xi \sigma} \partial_{t}\left(e^{2 \sigma \varphi(x, t, r)}\right) \leq-\frac{C}{\sigma} \partial_{t}\left(e^{2 \sigma \varphi(x, t, r)}\right)$.

Therefore,

$$
\begin{aligned}
\int_{\widetilde{Q}}\left(\int_{-T / 2}^{t}|u(x, s, r)| d s\right)^{2} e^{2 \sigma \varphi(x, t, r)} d x d t d r \\
\quad \leq \int_{\Omega} \int_{-3 \eta}^{3 \eta} \int_{-T / 2}^{5 T / 2}-\frac{C}{\sigma}\left(\int_{-T / 2}^{t}|u(x, s, r)|^{2} d s\right) \partial_{t}\left(e^{2 \sigma \varphi(x, t, r)}\right) d t d x d r \\
\quad \leq \frac{C}{\sigma} \int_{\Omega} \int_{-3 \eta}^{3 \eta}\left[\int_{-T / 2}^{5 T / 2}|u(x, t, r)|^{2} e^{2 \sigma \varphi(x, t, r)} d t d x d r\right. \\
\left.\quad-e^{2 \sigma \varphi(x, 5 T / 2, r)}\left(\int_{-T / 2}^{5 T / 2}|u(x, s, r)|^{2} d s\right) d x d r\right]
\end{aligned}
$$


Thus, we deduce the result.

We are now ready to state the Carleman estimate for the operator $\mathcal{Q}$ :

Theorem 4 (Carleman estimate) Let $\mathcal{Q}$ be the operator defined by (24). Let $K$ be a compact set in $\bar{\Omega} \times\left(-\frac{T}{2}, \frac{5 T}{2}\right) \times(-3 \eta, 3 \eta)$. We assume that

(H1) $(\lambda, \mu) \in C^{2}(\bar{\Omega})^{2}$ and $(\tilde{\lambda}, \tilde{\mu}) \in C^{2}(\overline{\Omega \times(0,+\infty)})^{2}$.

Then, there exists $\xi_{0}>0$ such that, for all $\xi \geq \xi_{0}$, there exists $\sigma_{0}>0$ such that, for all $\sigma \geq \sigma_{0}$ and for all $u \in C_{0}^{\infty}(K)^{3}$, we have the following estimate:

$$
\frac{1}{\sigma}\left\|u e^{\sigma \varphi}\right\|_{H^{2, \sigma}(\widetilde{Q})}^{2} \leq C\left(\left\|(\mathcal{Q} u) e^{\sigma \varphi}\right\|_{H^{1}(\widetilde{Q})}^{2}+\left\|u e^{\sigma \varphi}\right\|_{H^{2, \sigma}(\widetilde{\Sigma})}^{2}\right),
$$

where $\varphi$ is defined by (28), and $C>0$ depends on the $C^{2}(\bar{Q})$-norm of the coefficient $\tilde{\mu}$ but is independent of $\sigma$.

Proof Let $K$ be a compact set in $\bar{\Omega} \times\left(-\frac{T}{2}, \frac{5 T}{2}\right) \times(-3 \eta, 3 \eta)$, let $u \in C_{0}^{\infty}(K)^{3}$ be the solution of:

$$
\mathcal{Q} u(x, t, r)=S(x, t, r), \quad \forall(x, t, r) \in \widetilde{Q},
$$

and let assume (H1). We must first decouple the equations. To this end, we take the divergence of (29) and we introduce the scalar $v=\nabla \cdot u$ and the vector $w=\nabla \wedge u$. We obtain the following system of seven scalar equations only coupled at the first order:

$$
\begin{aligned}
-2 \partial_{r}^{2} u(x, t, r)- & \partial_{t}^{2} u(x, t, r)-\mu(x) \Delta u(x, t, r)+\int_{-T / 2}^{t} \tilde{\mu}(x, t-s) \Delta u(x, s, r) d s \\
& =S(x, t, r)+A_{1}(u, v)(x, t, r), \quad \forall(x, t, r) \in \widetilde{Q}, \\
-2 \partial_{r}^{2} v(x, t, r)- & \partial_{t}^{2} v(x, t, r)-(\lambda+2 \mu)(x) \Delta v(x, t, r) \\
& +\int_{-T / 2}^{t}(\tilde{\lambda}+2 \tilde{\mu})(x, t-s) \Delta v(x, s, r) d s \\
& =(\nabla \cdot S)(x, t, r)+A_{2}(u, v, w)(x, t, r), \quad \forall(x, t, r) \in \widetilde{Q}, \\
-2 \partial_{r}^{2} w(x, t, r)- & \partial_{t}^{2} w(x, t, r)-\mu(x) \Delta w(x, t, r)+\int_{-T / 2}^{t} \tilde{\mu}(x, t-s) \Delta w(x, s, r) d s \\
& =(\nabla \wedge S)(x, t, r)+A_{3}(u, v, w)(x, t, r), \quad \forall(x, t, r) \in \widetilde{Q},
\end{aligned}
$$

where $A_{1}, A_{2}$ and $A_{3}$ are first order integrodifferential operators. We only treat the first equation of (30), the others equations can be treated accordingly. We introduce the following change of variable:

$\tilde{u}(x, t, r)=\mu(x) u(x, t, r)-\int_{-T / 2}^{t} \tilde{\mu}(x, t-s, r) u(x, s, r) d s, \quad \forall(x, t, r) \in \widetilde{Q}$.

Thus, $\tilde{u}$ satisfies the classical elliptic equation

$$
\begin{aligned}
&-2 \partial_{r}^{2} \tilde{u}(x, t, r)-\partial_{t}^{2} \tilde{u}(x, t, r)-\mu(x) \Delta \tilde{u}(x, t, r) \\
&=\mu(x)\left(S(x, t, r)+A_{1}(u, v)(x, t, r)\right)+L_{1}(u)(x, t, r),
\end{aligned}
$$

where $L_{1}$ is a first order integro-differential operator with bounded coefficients. We now need a Carleman estimate for a scalar elliptic equation. It is given by the following lemma: 
Lemma 7 ([28] and [29]) Let $Q$ be an open domain in $\mathbb{R}^{n}, K$ be a compact set in $\bar{Q}$ and $\psi$ be a $C^{\infty}(Q)$ function satisfying $\nabla \psi(x) \neq 0, \forall x \in K$. Let

$$
\varphi(x)=e^{-\xi \psi(x)}, \quad \forall x \in Q,
$$

where $\xi>0$ is sufficiently large. We consider the scalar second-order elliptic operator

$$
\mathcal{R}(x) \star=a(x): \nabla^{2} \star+b(x) \cdot \nabla \star+c \star, \quad \forall x \in Q,
$$

where all the coefficients are $C^{2}(\bar{Q})$. Then, there exist $\sigma_{0}>0$ and $C>0$ such that, for all $\sigma \geq \sigma_{0}$ and for all $u \in C_{0}^{\infty}(K)$, the following Carleman estimates hold true:

$$
\begin{aligned}
& \frac{C}{\sigma}\left\|u e^{\sigma \varphi}\right\|_{H^{2, \sigma}(Q)}^{2} \leq\left\|(\mathcal{R} u) e^{\sigma \varphi}\right\|_{L^{2}(Q)}^{2}+\left\|u e^{\sigma \varphi}\right\|_{H^{2, \sigma}(\partial Q)}^{2}, \\
& C \sigma\left\|u e^{\sigma \varphi}\right\|_{H^{1, \sigma}(Q)}^{2} \leq\left\|(\mathcal{R} u) e^{\sigma \varphi}\right\|_{L^{2}(Q)}^{2}+\sigma\left\|u e^{\sigma \varphi}\right\|_{H^{1, \sigma}(\partial Q)}^{2} .
\end{aligned}
$$

As the weight function $\varphi$ defined in (28) satisfies the hypothesis of Lemma 7, we can apply the first inequality to $\tilde{u}$ :

$\frac{1}{\sigma}\left\|\tilde{u} e^{\sigma \varphi}\right\|_{H^{2, \sigma}(\widetilde{Q})}^{2} \leq C\left(\left\|\left(-2 \partial_{r}^{2} \tilde{u}-\partial_{t}^{2} \tilde{u}-\mu \Delta \tilde{u}\right) e^{\sigma \varphi}\right\|_{L^{2}(\widetilde{Q})}^{2}+\left\|\tilde{u} e^{\sigma \varphi}\right\|_{H^{2, \sigma}(\widetilde{\Sigma})}^{2}\right)$.

We take into account the fact that the coefficients $\lambda, \tilde{\lambda}, \mu, \tilde{\mu}$ are bounded in $Q$, that the Lemma 6 holds and that all the terms of the operators $A_{1}$ and $L_{1}$ are of order less or equal than 1 . Then, we obtain

$$
\begin{aligned}
& \frac{1}{\sigma}\left\|\tilde{u} e^{\sigma \varphi}\right\|_{H^{2, \sigma}(\widetilde{Q})}^{2} \\
& \quad \leq C\left(\left\|S e^{\sigma \varphi}\right\|_{L^{2}(\widetilde{Q})}^{2}+\left\|u e^{\sigma \varphi}\right\|_{H^{1, \sigma}(\widetilde{Q})}^{2}+\left\|v e^{\sigma \varphi}\right\|_{H^{1, \sigma}(\widetilde{Q})}^{2}+\left\|u e^{\sigma \varphi}\right\|_{H^{2, \sigma}(\widetilde{\Sigma})}^{2}\right) .
\end{aligned}
$$

We use again the change of variable

$$
u(x, t, r)=\frac{1}{\mu(x)} \tilde{u}(x, t, r)+\int_{-T / 2}^{t} \frac{\tilde{\mu}(x, t-s)}{\mu(x)} u(x, s, r) d s, \quad \forall(x, t, r) \in \widetilde{Q} .
$$

Hence,

$$
\begin{aligned}
& \int_{\widetilde{Q}}|u(x, t, r)|^{2} e^{2 \sigma \varphi(x, t, r)} d x d t d r \\
& \quad \leq C \int_{\widetilde{Q}}|\tilde{u}(x, t, r)|^{2} e^{2 \sigma \varphi(x, t, r)} d x d t d r+\frac{C}{\sigma} \int_{\widetilde{Q}}|u(x, t, r)|^{2} e^{2 \sigma \varphi(x, t, r)} d x d t d r .
\end{aligned}
$$

For $\sigma$ sufficiently large, the second term is absorbed. We do the same for the derivatives of $u$, which leads to

$$
\frac{1}{\sigma}\left\|u e^{\sigma \varphi}\right\|_{H^{2, \sigma}(\widetilde{Q})}^{2} \leq C \frac{1}{\sigma}\left\|\tilde{u} e^{\sigma \varphi}\right\|_{H^{2, \sigma}(\widetilde{Q})}^{2} .
$$

Finally,

$$
\begin{aligned}
& \frac{1}{\sigma}\left\|u e^{\sigma \varphi}\right\|_{H^{2, \sigma}(\widetilde{Q})}^{2} \\
& \quad \leq C\left(\left\|S e^{\sigma \varphi}\right\|_{L^{2}(\widetilde{Q})}^{2}+\left\|u e^{\sigma \varphi}\right\|_{H^{1, \sigma}(\widetilde{Q})}^{2}+\left\|v e^{\sigma \varphi}\right\|_{H^{1, \sigma}(\widetilde{Q})}^{2}+\left\|u e^{\sigma \varphi}\right\|_{H^{2, \sigma}(\widetilde{\Sigma})}^{2}\right) .
\end{aligned}
$$


Thanks to the Carleman weights, we absorb the second term of the right hand side in the left hand side. We make the same work for $v$ and $w$ but using the second Carleman inequality of the Lemma 7 in order to obtain

$$
\begin{aligned}
& \sigma\left\|v e^{\sigma \varphi}\right\|_{H^{1, \sigma}(\widetilde{Q})}^{2} \\
& \leq C\left(\left\|(\nabla \cdot S) e^{\sigma \varphi}\right\|_{L^{2}(\widetilde{Q})}^{2}+\left\|u e^{\sigma \varphi}\right\|_{H^{1, \sigma}(\widetilde{Q})}^{2}+\left\|w e^{\sigma \varphi}\right\|_{H^{1, \sigma}(\widetilde{Q})}^{2}+\sigma\left\|v e^{\sigma \varphi}\right\|_{H^{1, \sigma}(\widetilde{\Sigma})}^{2}\right) \\
& \sigma\left\|w e^{\sigma \varphi}\right\|_{H^{1, \sigma}(\widetilde{Q})}^{2} \\
& \leq C\left(\left\|(\nabla \wedge S) e^{\sigma \varphi}\right\|_{L^{2}(\widetilde{Q})}^{2}+\left\|u e^{\sigma \varphi}\right\|_{H^{1, \sigma}(\widetilde{Q})}^{2}+\left\|v e^{\sigma \varphi}\right\|_{H^{1, \sigma}(\widetilde{Q})}^{2}+\sigma\left\|w e^{\sigma \varphi}\right\|_{H^{1, \sigma}(\widetilde{\Sigma})}^{2}\right) .
\end{aligned}
$$

Hence,

$$
\begin{aligned}
& \sigma\left\|v e^{\sigma \varphi}\right\|_{H^{1, \sigma}(\widetilde{Q})}^{2} \\
& \leq C\left(\left\|(\nabla S) e^{\sigma \varphi}\right\|_{L^{2}(\widetilde{Q})}^{2}+\left\|u e^{\sigma \varphi}\right\|_{H^{1, \sigma}(\widetilde{Q})}^{2}+\left\|w e^{\sigma \varphi}\right\|_{H^{1, \sigma}(\widetilde{\Sigma})}^{2}+\sigma\left\|v e^{\sigma \varphi}\right\|_{H^{1, \sigma}(\widetilde{\Sigma})}^{2}\right) .
\end{aligned}
$$

Finally,

$$
\begin{aligned}
& \frac{1}{\sigma}\left\|u e^{\sigma \varphi}\right\|_{H^{2, \sigma}(\widetilde{Q})}^{2} \\
& \leq C\left(\left\|Q u e^{\sigma \varphi}\right\|_{L^{2}(\widetilde{Q})}^{2}+\frac{1}{\sigma}\left\|(\nabla Q u) e^{\sigma \varphi}\right\|_{L^{2}(\widetilde{Q})}^{2}+\frac{1}{\sigma}\left\|u e^{\sigma \varphi}\right\|_{H^{1, \sigma}(\widetilde{Q})}^{2}+\left\|u e^{\sigma \varphi}\right\|_{H^{2, \sigma}(\widetilde{\Sigma})}^{2}\right) .
\end{aligned}
$$

And thanks to the Carleman weights, we absorb the third term of the right hand side in the left hand side to obtain the result:

$$
\frac{1}{\sigma}\left\|u e^{\sigma \varphi}\right\|_{H^{2, \sigma}(\widetilde{Q})}^{2} \leq C\left(\left\|Q u e^{\sigma \varphi}\right\|_{H^{1}(\widetilde{Q})}^{2}+\left\|u e^{\sigma \varphi}\right\|_{H^{2, \sigma}(\widetilde{\Sigma})}^{2}\right)
$$

and this concludes the proof of Theorem 4 .

\subsection{Obtain local estimations}

We are now going to apply the Carleman estimate we proved in Theorem 4 to the function $u_{\gamma}$. To ensure that its support is in a compact set of $\bar{\Omega} \times\left(-\frac{T}{2}, \frac{5 T}{2}\right) \times(-3 \eta, 3 \eta)$, we multiply it by a cut-off function. A good choice of this cut-off function leads to two local estimations. Summing these estimations, we obtain the desired interpolation result. This work is inspired from [23] which uses some results of [24] but noticing that we have here an additional variable.

\subsection{First estimation}

Let $\Gamma \subset \partial \Omega$ be arbitrarily small. We begin by estimating $u_{\gamma}$ in a ball, which is close to $\Gamma$. Let us choose $0<\eta<\varepsilon$ and $x^{(0)} \in \mathbb{R}^{3} \backslash \bar{\Omega}$ such that

$$
\overline{B\left(x^{(0)}, \eta\right)} \cap \bar{\Omega}=\emptyset, \quad B\left(x^{(0)}, 2 \eta\right) \cap \Omega \neq \emptyset, \quad B\left(x^{(0)}, 4 \eta\right) \cap \partial \Omega \subset \Gamma .
$$

Figure 10 illustrates this construction. We define then

$$
\psi^{(0)}(x, t, r)=\left|x-x^{(0)}\right|^{2}+\frac{\eta^{2}}{T^{2}}\left(t+\frac{T}{2}\right)^{2}+r^{2}, \quad \forall(x, t, r) \in \widetilde{Q},
$$

and we set

$$
\varphi^{(0)}(x, t, r)=e^{-\frac{\xi}{\eta^{2}} \psi^{(0)}(x, t, r)}, \quad \forall(x, t, r) \in \widetilde{Q} .
$$




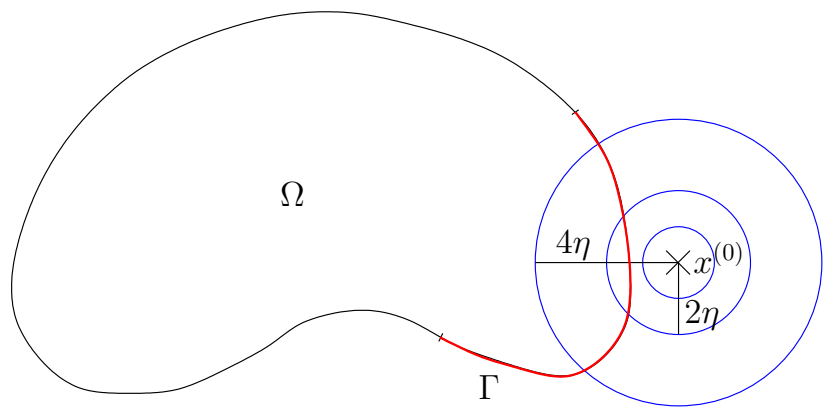

Figure 10. Definition of $x^{(0)}$ and $\eta$.

As in Figure 11, we introduce the cut-off function $\chi_{4} \in C_{0}^{\infty}(\mathbb{R})$ such that

$$
\chi_{4}(z)= \begin{cases}0, & \text { if } \quad z<\frac{1}{2}, z>8, \\ 1, & \text { if } \quad \frac{3}{4}<z<7\end{cases}
$$

and we set

$$
u_{\gamma}^{*}(x, t, r)=\chi_{4}\left(\frac{\psi^{(0)}}{\eta^{2}}\right) u_{\gamma}(x, t, r), \quad \forall(x, t, r) \in \widetilde{Q} .
$$

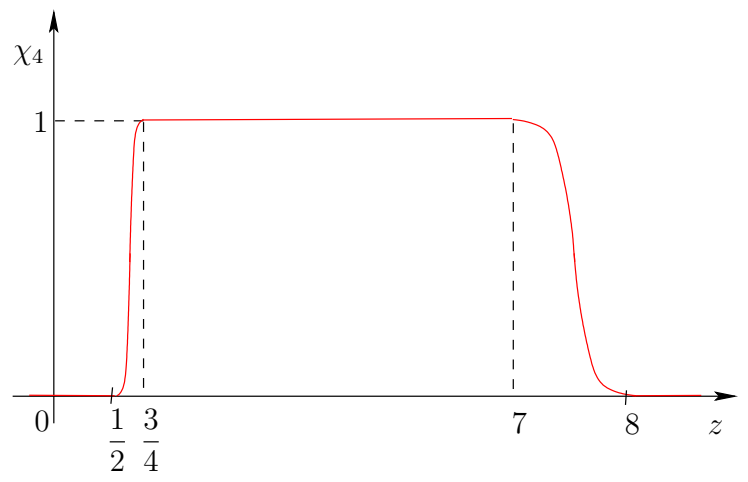

Figure 11. Definition of the cut-off function $\chi_{4}$.

Here, $u_{\gamma}^{*}$ has a compact support in $\bar{\Omega} \times\left(-\frac{T}{2}, \frac{5 T}{2}\right) \times(-3 \eta, 3 \eta)$ since

$$
\begin{aligned}
& \psi^{(0)}(x, t, \pm 3 \eta)>10 \eta^{2} \quad \Longrightarrow \quad u_{\gamma}^{*}(x, t, r)=0 \quad \text { if } \quad|r| \geq 3 \eta, \\
& \psi^{(0)}\left(x, \frac{5 T}{2}, r\right)>10 \eta^{2} \quad \Longrightarrow \quad u_{\gamma}^{*}(x, t, r)=0 \quad \text { if } \quad t \geq \frac{5 T}{2},
\end{aligned}
$$

and

$$
u_{\gamma}(x, t, r)=0 \quad \text { if } \quad t<-\frac{T}{2}+\frac{3 \eta}{2} .
$$

Therefore, we can apply the Carleman estimate we showed in Theorem 4 to this function:

$\frac{1}{\sigma}\left\|u_{\gamma}^{*} e^{\sigma \varphi^{(0)}}\right\|_{H^{2, \sigma}(\widetilde{Q})}^{2} \leq C\left(\left\|\left(\mathcal{Q} u_{\gamma}^{*}\right) e^{\sigma \varphi^{(0)}}\right\|_{H^{1}(\widetilde{Q})}^{2}+\left\|u_{\gamma}^{*} e^{\sigma \varphi^{(0)}}\right\|_{H^{2, \sigma}(\widetilde{\Sigma})}^{2}\right)$. 
The last norm is actually in $\widetilde{\Gamma}=\Gamma \times\left(-\frac{T}{2}, \frac{5 T}{2}\right) \times(-3 \eta, 3 \eta) \subset \widetilde{\Sigma}$. Indeed, we supposed $B\left(x^{(0)}, 4 \eta\right) \cap \partial \Omega \subset \Gamma$, therefore, if $x \in \partial \Omega \backslash \Gamma$, then $u_{\gamma}^{*}(x, t, r)=0$. In addition, $\forall(x, t, r) \in \widetilde{Q}$,

$\mathcal{Q} u_{\gamma}^{*}(x, t, r)$

$$
\begin{aligned}
& =\chi_{4}\left(\frac{\psi^{(0)}}{\eta^{2}}\right) \mathcal{Q} u_{\gamma}(x, t, r)+\left[\mathcal{Q}, \chi_{4}\left(\frac{\psi^{(0)}}{\eta^{2}}\right)\right] u_{\gamma}(x, t, r) \\
& =\chi_{4}\left(\frac{\psi^{(0)}}{\eta^{2}}\right)\left(F_{\gamma}(x, t, r)+G_{\gamma}(x, t, r)\right)+\left[\mathcal{Q}, \chi_{4}\left(\frac{\psi^{(0)}}{\eta^{2}}\right)\right] u_{\gamma}(x, t, r) \\
& =\chi_{4}\left(\frac{\psi^{(0)}}{\eta^{2}}\right) F_{\gamma}(x, t, r)+\left[\mathcal{Q}, \chi_{4}\left(\frac{\psi^{(0)}}{\eta^{2}}\right)\right] u_{\gamma}(x, t, r),
\end{aligned}
$$

since we saw in $(25)$ that $G_{\gamma}(x, t, r)=0, \forall x \in \Omega \backslash \overline{\Omega(3 \varepsilon)}$. And here, $\chi_{4}\left(\frac{\psi^{(0)}}{\eta^{2}}\right)$ is different from zero if $\left|x-x^{(0)}\right|^{2} \leq 8 \eta^{2}$, thus, in particular, if $\left|x-x^{(0)}\right| \leq 3 \eta<3 \varepsilon$. Then, we can write:

$$
\begin{aligned}
& \frac{1}{\sigma}\left\|u_{\gamma}^{*} e^{\sigma \varphi^{(0)}}\right\|_{H^{2, \sigma}(\widetilde{Q})}^{2} \leq C\left(\left\|\left(\mathcal{Q} u_{\gamma}^{*}\right) e^{\sigma \varphi^{(0)}}\right\|_{H^{1}(\widetilde{Q})}^{2}+\left\|u_{\gamma}^{*} e^{\sigma \varphi^{(0)}}\right\|_{H^{2, \sigma}(\widetilde{\Gamma})}^{2}\right) \\
& \Longrightarrow \frac{1}{\sigma} e^{2 \sigma e^{-6 \xi}}\left\|u_{\gamma}\right\|_{H^{2, \sigma}\left(\left\{\eta^{2} \leq \psi^{(0)} \leq 6 \eta^{2}\right\} \cap \widetilde{Q}\right)}^{2} \\
& \quad \leq C\left(\sigma^{2} e^{2 \sigma e^{-7 \xi}}\left\|u_{\gamma}\right\|_{H^{2}(\widetilde{Q})}^{2}+\sigma^{2} e^{2 \sigma e^{-\frac{\xi}{2}}}\left\|F_{\gamma}\right\|_{H^{1}(\widetilde{Q})}^{2}+\sigma^{4} e^{2 \sigma e^{-\frac{\xi}{2}}}\left\|u_{\gamma}\right\|_{H^{2}(\widetilde{\Gamma})}^{2}\right),
\end{aligned}
$$

We choose then $\rho$ and $x^{(1)}$ such that $\operatorname{dist}\left(x^{(1)}, \partial \Omega\right) \geq 4 \rho, \quad \widetilde{B}_{1}=B\left(x^{(1)}, \rho\right) \times\left(-\frac{T}{2}, \frac{T}{2}\right) \times(-\rho, \rho) \subset\left\{\eta^{2} \leq \psi^{(0)} \leq 6 \eta^{2}\right\}$.

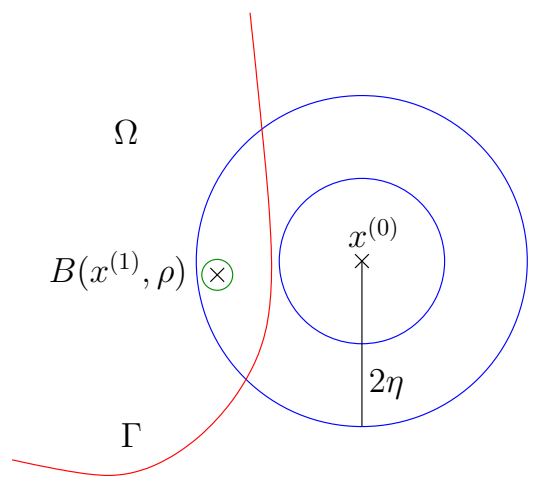

Figure 12. Definition of $x^{(1)}$ and $\rho$.

This choice is valid since, in $\widetilde{B}_{1}$, we have

$$
\begin{aligned}
\psi^{(0)}(x, t, r) & \leq\left|x-x^{(1)}\right|^{2}+\left|x^{(1)}-x^{(0)}\right|^{2}+\frac{\eta^{2}}{T^{2}}\left(t+\frac{T}{2}\right)^{2}+r^{2} \\
& \leq \rho^{2}+\left|x^{(1)}-x^{(0)}\right|^{2}+\eta^{2}+\rho^{2}
\end{aligned}
$$

and since $B\left(x^{(0)}, 2 \eta\right) \cap \Omega \neq \emptyset$, we can choose $x^{(1)}$ such that $\left|x^{(1)}-x^{(0)}\right|^{2}<4 \eta^{2}$ and $4 \rho \leq \eta$ so that $\psi^{(0)}(x, t, r) \leq 6 \eta^{2}$. Then, if $\sigma$ is sufficiently large, we have $\left\|u_{\gamma}\right\|_{H^{2}\left(\widetilde{B}_{1}\right)}^{2} \leq C e^{-C_{1} \sigma}\left\|u_{\gamma}\right\|_{H^{2}(\widetilde{Q})}^{2}+C e^{C_{2} \sigma}\left(\left\|F_{\gamma}\right\|_{H^{1}(\widetilde{Q})}^{2}+\left\|u_{\gamma}\right\|_{H^{2}(\widetilde{\Gamma})}^{2}\right)$. 
We minimize with respect to $\sigma$ (cf. [24]) to obtain, with $\nu_{0}=\frac{C_{2}}{C_{1}+C_{2}}$ :

$$
\left\|u_{\gamma}\right\|_{H^{2}\left(\widetilde{B}_{1}\right)} \leq C\left(\left\|u_{\gamma}\right\|_{H^{2}(\widetilde{Q})}\right)^{1-\nu_{0}}\left(\left\|F_{\gamma}\right\|_{H^{1}(\widetilde{Q})}+\left\|u_{\gamma}\right\|_{H^{2}(\widetilde{\Gamma})}\right)^{\nu_{0}} .
$$

\subsection{Second estimation}

Now, we extend the estimation in $B\left(x^{(1)}, \rho\right)$ into $\Omega(\varepsilon) \backslash \Omega(2 \varepsilon)$. Let $B\left(x^{(j)}, \rho\right)$, $2 \leq j \leq N$ be a cover of $\Omega(\varepsilon) \backslash \Omega(2 \varepsilon)$. We suppose that $x^{(j)}$ is such that for $2 \leq j \leq N$, $\operatorname{dist}\left(x^{(j)}, \partial \Omega\right) \geq 4 \rho, \quad$ and $B\left(x^{(j+1)}, \rho\right) \subset B\left(x^{(j)}, 2 \rho\right)$.
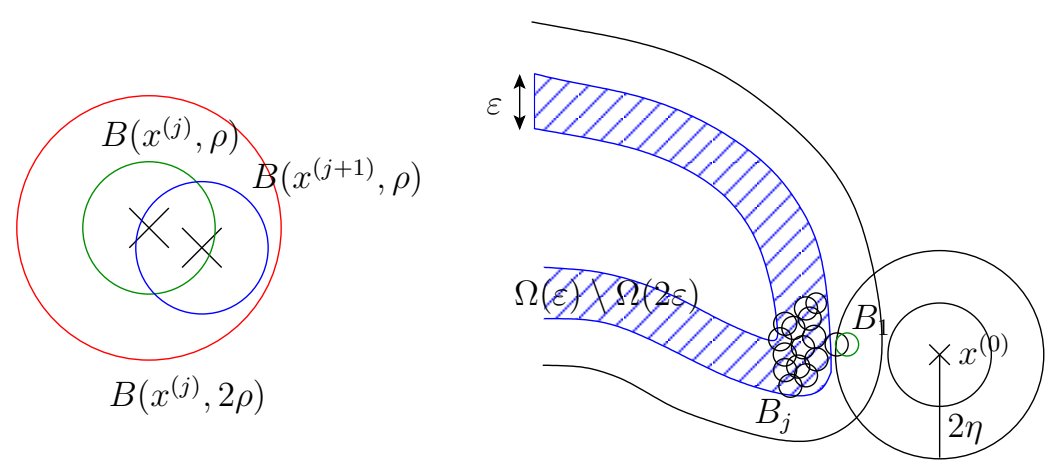

Figure 13. Definition of the balls $B\left(x^{(j)}, \rho\right)$.

We introduce

$$
\widetilde{B}_{j}=B\left(x^{(j)}, \rho\right) \times\left(-\frac{T}{2}, \frac{T}{2}\right) \times(-\rho, \rho) .
$$

This construction is illustrated in Figure 13. We define

$$
\psi^{(j)}(x, t, r)=\left|x-x^{(j)}\right|^{2}+\frac{\rho^{2}}{T^{2}}\left(t+\frac{T}{2}\right)^{2}+r^{2}, \quad \forall(x, t, r) \in \widetilde{Q},
$$

and

$$
\varphi^{(j)}(x, t, r)=e^{-\frac{\xi}{\rho^{2}} \psi^{(j)}(x, t, r)}, \quad \forall(x, t, r) \in \widetilde{Q} .
$$

We set

$$
u_{\gamma}^{*}(x, t, r)=\chi_{4}\left(\frac{\psi^{(j)}}{\rho^{2}}\right) u_{\gamma}(x, t, r), \quad \forall(x, t, r) \in \widetilde{Q},
$$

to which we apply Theorem 4 , without the boundary term, since the support of $u_{\gamma}^{*}$ is an interior domain:

$$
\frac{1}{\sigma}\left\|u_{\gamma}^{*} e^{\sigma \varphi^{(j)}}\right\|_{H^{2, \sigma}(\widetilde{Q})}^{2} \leq C\left\|\left(\mathcal{Q} u_{\gamma}^{*}\right) e^{\sigma \varphi^{(j)}}\right\|_{H^{1}(\widetilde{Q})}^{2} .
$$

In addition, $\forall(x, t, r) \in \widetilde{Q}$,

$$
\mathcal{Q} u_{\gamma}^{*}(x, t, r)=\chi_{4}\left(\frac{\psi^{(j)}}{\rho^{2}}\right) F_{\gamma}(x, t, r)+\left[\mathcal{Q}, \chi_{4}\left(\frac{\psi^{(j)}}{\rho^{2}}\right)\right] u_{\gamma}(x, t, r) .
$$


Thus, we have

$$
\begin{aligned}
& \frac{C}{\sigma} e^{2 \sigma e^{-6 \xi}}\left\|u_{\gamma}\right\|_{H^{2, \sigma}\left(\left\{\rho^{2} \leq \psi^{(j)} \leq 6 \rho^{2}\right\} \cap \widetilde{Q}\right)}^{2} \\
& \leq \sigma^{2} e^{2 \sigma e^{-\frac{\xi}{2}}}\left\|u_{\gamma}\right\|_{H^{2}\left(\psi^{(j)} \leq \rho^{2}\right)}^{2}+\sigma^{2} e^{2 \sigma e^{-7 \xi}}\left\|u_{\gamma}\right\|_{H^{2}\left(\psi^{(j)} \leq 8 \rho^{2}\right)}^{2}+\sigma^{2} e^{2 \sigma e^{-\frac{\xi}{2}}}\left\|F_{\gamma}\right\|_{H^{1}(\widetilde{Q})}^{2},
\end{aligned}
$$

and, if we choose $\sigma$ large, we can write:

$$
\begin{aligned}
& e^{2 \sigma e^{-6 \xi}}\left\|u_{\gamma}\right\|_{H^{2, \sigma}\left(\left\{\psi^{(j)} \leq 6 \rho^{2}\right\} \cap \widetilde{Q}\right)}^{2} \\
& \leq C e^{2 \sigma e^{-\frac{\xi}{3}}}\left\|u_{\gamma}\right\|_{H^{2}\left(\psi^{(j)} \leq \rho^{2}\right)}^{2}+C e^{2 \sigma e^{-\frac{13 \xi}{2}}}\left\|u_{\gamma}\right\|_{H^{2}\left(\psi^{(j)} \leq 8 \rho^{2}\right)}^{2}+C e^{2 \sigma e^{-\frac{\xi}{3}}}\left\|F_{\gamma}\right\|_{H^{1}(\widetilde{Q})}^{2} .
\end{aligned}
$$

This allows to conclude that

$\left\|u_{\gamma}\right\|_{H^{2}\left(\widetilde{B}_{j+1}\right)}^{2} \leq C e^{C_{3} \sigma}\left(\left\|u_{\gamma}\right\|_{H^{2}\left(\widetilde{B}_{j}\right)}^{2}+\left\|F_{\gamma}\right\|_{H^{1}(\widetilde{Q})}^{2}\right)+C e^{-C_{4} \sigma}\left\|u_{\gamma}\right\|_{H^{2}(\widetilde{Q})}^{2}$,

since $\widetilde{B}_{j+1} \subset\left\{\psi^{(j)} \leq 6 \rho^{2}\right\}$ and $\left\{\psi^{(j)} \leq \rho^{2}\right\} \subset \widetilde{B}_{j}$. We minimize with respect to $\sigma$ to obtain, with $\nu_{1}=\frac{C_{4}}{C_{3}+C_{4}}$ :

$$
\left\|u_{\gamma}\right\|_{H^{2}\left(\widetilde{B}_{j+1}\right)} \leq C\left(\left\|u_{\gamma}\right\|_{H^{2}(\widetilde{Q})}\right)^{1-\nu_{1}}\left(\left\|F_{\gamma}\right\|_{H^{1}(\widetilde{Q})}+\left\|u_{\gamma}\right\|_{H^{2}\left(\widetilde{B}_{j}\right)}\right)^{\nu_{1}}
$$

We use then the recurrence result of the following lemma:

Lemma 8 (Lemma 4 in [29]) Let $\alpha_{j}>0$ satisfying, for all $j \geq 0$,

$$
\alpha_{j} \leq B^{1-\nu}\left(\alpha_{j-1}+A\right)^{\nu} \quad \text { and } \quad \alpha_{j} \leq B
$$

where $A>0, B>0$ and $\nu \in] 0,1[$. Then, for all $\mu \in] 0, \nu^{N}$ [, we have:

$$
\alpha_{N} \leq 2^{1 /(1-\nu)} B^{1-\mu}\left(\alpha_{0}+A\right)^{\mu} .
$$

Here,

$$
\alpha_{j}=\left\|u_{\gamma}\right\|_{H^{2}\left(\widetilde{B}_{j+1}\right)}, \quad A=\left\|F_{\gamma}\right\|_{H^{1}(\widetilde{Q})}, \quad B=\left\|u_{\gamma}\right\|_{H^{2}(\widetilde{Q})}^{2} .
$$

Therefore, we obtain

$$
\left\|u_{\gamma}\right\|_{H^{2}\left(\widetilde{B}_{n}\right)} \leq C\left(\left\|u_{\gamma}\right\|_{H^{2}(\widetilde{Q})}\right)^{1-\nu}\left(\left\|F_{\gamma}\right\|_{H^{1}(\widetilde{Q})}+\left\|u_{\gamma}\right\|_{H^{2}\left(\widetilde{B}_{1}\right)}\right)^{\nu} .
$$

We apply the Young inequality and we obtain

$$
\left\|u_{\gamma}\right\|_{H^{2}\left(\widetilde{B}_{n}\right)} \leq \epsilon^{q}\left\|u_{\gamma}\right\|_{H^{2}(\widetilde{Q})}+\epsilon^{-q^{\prime}}\left(\left\|F_{\gamma}\right\|_{H^{1}(\widetilde{Q})}+\left\|u_{\gamma}\right\|_{H^{2}\left(\widetilde{B}_{1}\right)}\right),
$$

where $q=\frac{1}{1-\nu}$ and $q^{\prime}=\frac{1}{\nu}$. We use the estimations (26) and (27) on $F_{\gamma}$ and $u_{\gamma}$ to write:

$\left\|u_{\gamma}\right\|_{H^{2}\left(\widetilde{B}_{n}\right)}$

$$
\leq \epsilon^{q} e^{M \gamma}\left\|u^{*}\right\|_{H^{2}(\Omega \times(0,3 T))}+\epsilon^{-q^{\prime}}\left(e^{-m \gamma T}\left\|u^{*}\right\|_{H^{2}(\Omega \times(0,3 T))}+\left\|u_{\gamma}\right\|_{H^{2}\left(\widetilde{B}_{1}\right)}\right) .
$$

We choose then $\epsilon=e^{-2 M \gamma / q}$, so that

$$
\begin{aligned}
\left\|u_{\gamma}\right\|_{H^{2}\left(\widetilde{B}_{n}\right)} \leq e^{-M \gamma}\left\|u^{*}\right\|_{H^{2}(\Omega \times(0,3 T))} & \\
& +e^{-\left(m T-2 M \frac{q^{\prime}}{q}\right) \gamma}\left\|u^{*}\right\|_{H^{2}(\Omega \times(0,3 T))}+e^{2 M \gamma \frac{q^{\prime}}{q}}\left\|u_{\gamma}\right\|_{H^{2}\left(\widetilde{B}_{1}\right)} .
\end{aligned}
$$

If we fix $T>T_{n}$ with

$$
m T_{n}-2 M \frac{q^{\prime}}{q}=M
$$


we obtain, with $\kappa=2 M \gamma \frac{q^{\prime}}{q}$,

$$
\left\|u_{\gamma}\right\|_{H^{2}\left(\widetilde{B}_{n}\right)} \leq e^{-M \gamma}\left\|u^{*}\right\|_{H^{2}(\Omega \times(0,3 T))}+e^{\kappa \gamma}\left\|u_{\gamma}\right\|_{H^{2}\left(\widetilde{B}_{1}\right)} .
$$

We can do the same work on the first estimation (31), the one in $\widetilde{B}_{1}$. We apply the Young inequality and the estimations (26) and (27) to obtain

$\left\|u_{\gamma}\right\|_{H^{2}\left(\widetilde{B}_{1}\right)}$

$$
\leq \epsilon^{q_{0}} e^{M \gamma}\left\|u^{*}\right\|_{H^{2}(\Omega \times(0,3 T))}+\epsilon^{-q_{0}^{\prime}}\left(e^{-m \gamma T}\left\|u^{*}\right\|_{H^{2}(\Omega \times(0,3 T))}+\left\|u_{\gamma}\right\|_{H^{2}(\widetilde{\Gamma})}\right) .
$$

where $q_{0}=\frac{1}{1-\nu_{0}}$ and $q_{0}^{\prime}=\frac{1}{\nu_{0}}$. We choose then $\epsilon=e^{-(2 M+\kappa) \gamma / q_{0}}$, so that

$$
\begin{aligned}
\left\|u_{\gamma}\right\|_{H^{2}\left(\widetilde{B}_{1}\right)} \leq e^{-(M+\kappa) \gamma}\left\|u^{*}\right\|_{H^{2}(\Omega \times(0,3 T))} & \\
& +e^{-\left(m T-(2 M+\kappa) \frac{q_{0}^{\prime}}{q_{0}}\right) \gamma}\left\|u^{*}\right\|_{H^{2}(\Omega \times(0,3 T))}+e^{(2 M+\kappa) \gamma \frac{q_{0}^{\prime}}{q_{0}}}\left\|u_{\gamma}\right\|_{H^{2}(\widetilde{\Gamma})} .
\end{aligned}
$$

If we fix $T>T_{0}$ with

$$
m T_{0}-(2 M+\kappa) \frac{q_{0}^{\prime}}{q_{0}}=M+\kappa,
$$

we obtain

$$
\left\|u_{\gamma}\right\|_{H^{2}\left(\widetilde{B}_{1}\right)} \leq e^{-(M+\kappa) \gamma}\left\|u^{*}\right\|_{H^{2}(\Omega \times(0,3 T))}+e^{C \gamma}\left\|u_{\gamma}\right\|_{H^{2}(\widetilde{\Gamma})} .
$$

And we regroup the two estimations in

$$
\left\|u_{\gamma}\right\|_{H^{2}\left(\widetilde{B}_{n}\right)} \leq e^{-M \gamma}\left\|u^{*}\right\|_{H^{2}(\Omega \times(0,3 T))}+e^{C \gamma}\left\|u^{*}\right\|_{H^{2}(\Gamma \times(0,3 T))}^{2} .
$$

Finally, we take $T>\max _{n} T_{n}$ and we sum the inequalities to obtain

$$
\left\|u_{\gamma}\right\|_{H^{2}\left(\Omega(\varepsilon) \backslash \Omega(2 \varepsilon) \times\left(-\frac{T}{2}, \frac{T}{2}\right) \times(-\rho, \rho)\right)}^{2} \leq e^{-C \gamma}\left\|u^{*}\right\|_{H^{2}(\Omega \times(0,3 T))}^{2}+e^{C \gamma}\left\|u^{*}\right\|_{H^{2}(\Gamma \times(0,3 T))}^{2} .
$$

We can now go back to the variable $u$ in the estimations.

\subsection{Return to the variable $u$}

We introduce the new variable

$$
\begin{aligned}
w_{\gamma}(x, t) & =u_{\gamma}(x, t, r=0)=\sqrt{\frac{\gamma}{2 \pi}} \int_{0}^{\infty} e^{-\frac{\gamma}{2}(t-y)^{2}} \theta(t-y) u^{*}(x, y) d y \\
& =\left(K_{\gamma} * u^{*}\right)(x, t), \quad \forall(x, t) \in \Omega \times \mathbb{R},
\end{aligned}
$$

with

$$
K_{\gamma}(t)=\sqrt{\frac{\gamma}{2 \pi}} e^{-\frac{\gamma}{2} t^{2}} \theta(t), \quad \forall t \in \mathbb{R} .
$$

We see that $w_{\gamma}$ converges to $u^{*}$ when $\gamma$ tends to infinity. We have

$$
\begin{aligned}
\left\|w_{\gamma}\right\|_{H^{2}\left(\Omega(\varepsilon) \backslash \Omega(2 \varepsilon) \times\left(0, \frac{T}{2}-\rho\right)\right)}^{2} & \leq C\left\|u_{\gamma}\right\|_{H^{2}\left(\Omega(\varepsilon) \backslash \Omega(2 \varepsilon) \times\left(-\frac{T}{2}, \frac{T}{2}\right) \times(-\rho, \rho)\right)}^{2} \\
& \leq e^{-C \gamma}\left\|u^{*}\right\|_{H^{2}(\Omega \times(0,3 T))}^{2}+e^{C \gamma}\left\|u^{*}\right\|_{H^{2}(\Gamma \times(0,3 T))}^{2} .
\end{aligned}
$$

Indeed, the Cauchy formula says that, for $0<d<\rho$, we have

$$
w_{\gamma}(x, a)=\frac{1}{2 i \pi} \int_{|w-a|=d} \frac{w_{\gamma}(x, w)}{w-a} d w
$$


which, using polar coordinates, implies that

$$
\left|w_{\gamma}(x, a)\right|^{2} \leq C \int_{0}^{2 \pi}\left|w_{\gamma}\left(x, a+d e^{i \theta}\right)\right|^{2} d \theta .
$$

We integrate then, for $0<d<\rho$, into

$$
\left|w_{\gamma}(x, a)\right|^{2} \leq \frac{C}{\rho} \int_{0}^{\rho} \int_{0}^{2 \pi}\left|w_{\gamma}\left(x, a+d e^{i \theta}\right)\right|^{2} d \theta d \rho,
$$

that we can write:

$$
\begin{aligned}
\left|w_{\gamma}(x, a)\right|^{2} & \leq C \int_{-\rho}^{\rho} \int_{|t-a| \leq \rho}\left|w_{\gamma}(x, t+i r)\right|^{2} d t d r \\
& =C \int_{-\rho}^{\rho} \int_{|t-a| \leq \rho}\left|u_{\gamma}(x, t, r)\right|^{2} d t d r
\end{aligned}
$$

according to the definition of $w_{\gamma}$. Then, we integrate for $x \in \Omega(\varepsilon) \backslash \Omega(2 \varepsilon)$ :

$$
\begin{aligned}
\left\|w_{\gamma}(., a)\right\|_{L^{2}(\Omega(\varepsilon) \backslash \Omega(2 \varepsilon))}^{2} & \leq C \int_{-\rho}^{\rho} \int_{|t-a| \leq \rho}\left\|u_{\gamma}(., t, r)\right\|_{L^{2}(\Omega(\varepsilon, 2 \varepsilon))}^{2} d t d r \\
& \leq C\left\|u_{\gamma}\right\|_{L^{2}\left(\Omega(\varepsilon, 2 \varepsilon) \times\left(-\frac{T}{2}, \frac{T}{2}\right) \times(-\rho, \rho)\right)}^{2} .
\end{aligned}
$$

We integrate for $a \in\left(0, \frac{T}{2}-\rho\right)$ :

$$
\left\|w_{\gamma}\right\|_{L^{2}\left(\Omega(\varepsilon) \backslash \Omega(2 \varepsilon) \times\left(0, \frac{T}{2}-\rho\right)\right)}^{2} \leq C\left\|u_{\gamma}\right\|_{L^{2}\left(\Omega(\varepsilon, 2 \varepsilon) \times\left(-\frac{T}{2}, \frac{T}{2}\right) \times(-\rho, \rho)\right)}^{2}
$$

and we do the same for the derivatives of $w_{\gamma}$. We can now come back to $u^{*}$ (and $u$ ) using the classical Fourier transform (noted with a big hat symbol) because we notice that

$$
w_{\gamma}(x, t)=\left(K_{\gamma} * u^{*}\right)(x, t) \Longrightarrow \widehat{w_{\gamma}}(x, \tau)=\widehat{K_{\gamma}}(\tau) \widehat{u^{*}}(x, \tau),
$$

where

$$
\begin{aligned}
\widehat{K_{\gamma}}(\tau) & =\sqrt{\frac{\gamma}{2 \pi}} \int_{-\infty}^{+\infty} e^{-i \tau t} e^{-\frac{\gamma}{2} t^{2}} \theta(t) d t \\
& =\sqrt{\frac{\gamma}{2 \pi}} \int_{-\infty}^{+\infty} e^{-i \tau t} e^{-\frac{\gamma}{2} t^{2}} d t+\sqrt{\frac{\gamma}{2 \pi}} \int_{-\infty}^{+\infty} e^{-i \tau t} e^{-\frac{\gamma}{2} t^{2}}(\theta(t)-1) d t
\end{aligned}
$$

We have

$$
\begin{aligned}
\sqrt{\frac{\gamma}{2 \pi}} \int_{-\infty}^{+\infty} e^{-i \tau t} e^{-\frac{\gamma}{2} t^{2}} d t & =\sqrt{\frac{\gamma}{2 \pi}} \int_{-\infty}^{+\infty} e^{-\left(\sqrt{\frac{\gamma}{2}} t+\sqrt{\frac{1}{2 \gamma}} i \tau\right)^{2}} e^{-\frac{\tau^{2}}{2 \gamma}} d t \\
& =\frac{1}{\sqrt{\pi}} e^{-\frac{\tau^{2}}{2 \gamma}} \int_{-\infty}^{+\infty} e^{-z^{2}} d z=e^{-\frac{\tau^{2}}{2 \gamma}}
\end{aligned}
$$

Hence,

$$
\widehat{K_{\gamma}}(\tau)=e^{-\frac{\tau^{2}}{2 \gamma}}+\sqrt{\frac{\gamma}{2 \pi}} \int_{-\infty}^{-\frac{T}{2}+3 \eta} e^{-i \tau t} e^{-\frac{\gamma}{2} t^{2}}(\theta(t)-1) d t .
$$

Therefore, we have

$$
\begin{aligned}
\left|\left(1-\widehat{K_{\gamma}}\right)(\tau)\right| & \leq\left|1-e^{-\frac{\tau^{2}}{2 \gamma}}\right|+\sqrt{\frac{\gamma}{2 \pi}} \int_{-\infty}^{-\frac{T}{2}+3 \eta}\left|e^{-i \tau t} e^{-\frac{\gamma}{2} t^{2}}\right||\theta(t)-1| d t \\
& \leq \frac{\tau^{2}}{2 \gamma}+\sqrt{\frac{\gamma}{2 \pi}} \int_{-\infty}^{-\frac{T}{2}+3 \eta} e^{-\frac{\gamma}{2} t^{2}} d t
\end{aligned}
$$


where we used for the first term the fact that the function $\frac{1-e^{-t^{2}}}{t^{2}}$ is bounded in $\mathbb{R}$. We win one order of convergence since we have $\frac{1}{\gamma}$ and no $\frac{1}{\sqrt{\gamma}}$ as in [14]. Then, we can introduce in the second term the change of variable $z=e^{-\frac{\gamma}{2} t^{2}}$, that is $t=\sqrt{-\frac{2}{\gamma} \ln (z)}$, and $d z=-\gamma t e^{-\frac{\gamma}{2} t^{2}} d t$, and so we have:

$$
\begin{aligned}
\sqrt{\frac{\gamma}{2 \pi}} \int_{-\infty}^{-\frac{T}{2}+3 \eta} e^{-\frac{\gamma}{2} t^{2}} d t & \leq \frac{1}{2 \sqrt{\pi}} \int_{0}^{e^{-\frac{\gamma}{2}\left(-\frac{T}{2}+3 \eta\right)^{2}}} \frac{d z}{\sqrt{-\ln (z)}} \\
& \leq \frac{1}{2 \sqrt{\pi}} \frac{e^{-\frac{\gamma}{2}\left(-\frac{T}{2}+3 \eta\right)^{2}}}{\sqrt{\frac{\gamma}{2}\left(-\frac{T}{2}+3 \eta\right)^{2}}} \leq \frac{C}{\gamma} .
\end{aligned}
$$

Then,

$$
\begin{aligned}
\left.\left\|u^{*}-w_{\gamma}\right\|_{L^{2}(\Omega(\varepsilon)}^{2} \backslash \Omega(2 \varepsilon) \times\left(0, \frac{T}{2}-\rho\right)\right) & \leq\left\|u^{*}-w_{\gamma}\right\|_{L^{2}(\Omega \times \mathbb{R})}^{2}=\left\|u^{*}-w_{\gamma}\right\|_{L^{2}(\Omega \times \mathbb{R})}^{2} \\
& \leq \int_{\Omega} \int_{-\infty}^{+\infty}\left|\frac{\tau^{2}+C}{\gamma}\right|^{2}\left|\widehat{u^{*}}(x, \tau)\right|^{2} d \tau \\
& \leq \frac{C}{\gamma^{2}} \int_{\Omega} \int_{-\infty}^{+\infty}\left(\left|\widehat{u^{*}}(x, \tau)\right|^{2}+\left|\widehat{\partial_{t}^{2} u^{*}}(x, \tau)\right|^{2}\right) d \tau \leq \frac{C}{\gamma^{2}}\left\|\widehat{u^{*}}\right\|_{H^{2}(\Omega \times \mathbb{R})}^{2} .
\end{aligned}
$$

Finally, if we suppose that $u^{*}$ is prolonged by zero outside $(0,3 T)$, we have

$$
\left\|u^{*}-w_{\gamma}\right\|_{L^{2}\left(\Omega(\varepsilon) \backslash \Omega(2 \varepsilon) \times\left(0, \frac{T}{2}-\rho\right)\right)} \leq \frac{C}{\gamma}\left\|u^{*}\right\|_{H^{2}(\Omega \times(0,3 T))} .
$$

Thus,

$$
\begin{aligned}
& \left\|u^{*}\right\|_{H^{2}\left(\Omega(\varepsilon) \backslash \Omega(2 \varepsilon) \times\left(0, \frac{T}{2}-\rho\right)\right)} \\
& \quad \leq C\left(\left\|u^{*}-w_{\gamma}\right\|_{H^{2}\left(\Omega(\varepsilon) \backslash \Omega(2 \varepsilon) \times\left(0, \frac{T}{2}-\rho\right)\right)}+\left\|w_{\gamma}\right\|_{H^{2}\left(\Omega(\varepsilon) \backslash \Omega(2 \varepsilon) \times\left(0, \frac{T}{2}-\rho\right)\right)}\right) \\
& \quad \leq \frac{C}{\gamma}\left\|u^{*}\right\|_{H^{4}(\Omega \times(0,3 T))}+C\left\|w_{\gamma}\right\|_{H^{2}\left(\Omega(\varepsilon) \backslash \Omega(2 \varepsilon) \times\left(0, \frac{T}{2}-\rho\right)\right)} .
\end{aligned}
$$

Coming back to $u$ and taking into account that $u^{*}(x, t)=\chi_{3}(x) u(x, t)$, so that $u=u^{*}$ in $\Omega(\varepsilon) \backslash \Omega(2 \varepsilon)$, we have

$\|u\|_{H^{2}\left(\Omega(\varepsilon) \backslash \Omega(2 \varepsilon) \times\left(0, \frac{T}{2}-\rho\right)\right)} \leq \frac{C}{\gamma}\|u\|_{H^{4}(\Omega \times(0,3 T))}+e^{C \gamma}\|u\|_{H^{2}(\Gamma \times(0,3 T))}$.

We use the fact that $\|u\|_{H^{4}(\Omega \times(0,3 T))}$ is bounded and that

$$
\|u\|_{H^{2}(\Gamma \times(0,3 T))}^{2}=\sum_{|\alpha|=1}^{2}\left\|\partial_{x}^{\alpha} u\right\|_{L^{2}(\Gamma \times(0,3 T))}^{2},
$$

since $\hat{u}$ and its time derivatives vanish on $\Gamma$, to write:

$$
\|u\|_{H^{2}\left(\Omega(\varepsilon) \backslash \Omega(2 \varepsilon) \times\left(0, \frac{T}{2}-\rho\right)\right)}^{2} \leq \frac{C_{5}}{\gamma^{2}}+e^{C_{6} \gamma} \sum_{|\alpha|=1}^{2}\left\|\partial_{x}^{\alpha} u\right\|_{L^{2}(\Gamma \times(0,3 T))}^{2} .
$$

Then, we choose $\gamma$ such that the first term dominate the second. We can for example choose

$$
\gamma=\frac{1}{2 C_{6}} \log \left(2+\frac{C_{5}}{\sum_{|\alpha|=1}^{2}\left\|\partial_{x}^{\alpha} u\right\|_{L^{2}(\Gamma \times(0,3 T))}^{2}}\right) .
$$

We achieve the proof of Theorem 3 . 


\section{Numerical results}

To illustrate the theoretical result (12), we present here one numerical example in 2D, even if the result is also valid in $3 \mathrm{D}$. The numerical resolution that we propose leads to recover the values of the unknown coefficient $p$ at each vertex of the discretized domain. Since the linear viscoelastic system (1) is a simplified model for the mechanical behavior of brain structures [30], we could use the method to retrieve the localization of a brain tumor. However, this example can be considered as an academic test case, sufficient to emphasize the motivation of the study, to illustrate the stability result and to propose an efficient numerical method.

\subsection{Direct problem}

We consider now the system (1)-(3), in two dimensions, with the following coefficients:

- $\mu(x)=\lambda(x)=1200, h(t)=e^{-t / \tau}$ with $\tau=1, \tilde{\lambda}(x, t)=400 h(t)$,

- $\bar{p}(x)= \begin{cases}400, & \text { in the healthy tissue, } \\ >400, & \text { in the tumor (cf. Figure 14), }\end{cases}$

- $f(x, t)=0, \bar{u}_{1}(x)=0$ and $\bar{u}_{0}$ is the solution of the stationary problem associated to $(1)$ :

$$
\begin{cases}-\nabla \cdot\left(\mu(x)\left(\nabla \bar{u}_{0}(x)+\nabla \bar{u}_{0}(x)^{T}\right)+\lambda(x)\left(\nabla \cdot \bar{u}_{0}\right)(x) I\right)=1, & \forall x \in \Omega, \\ u_{0}(x)=0, & \forall x \in \partial \Omega .\end{cases}
$$

When dealing with a real experiment, it is difficult to ensure that the initial data $\bar{u}_{0}$ satisfies hypothesis (H5). In practice, the recuperation is carry out with $\bar{u}_{0} \neq 0$. Moreover, we assume that $\bar{p}$ is known in the boundary of $\Omega$. This hypothesis, less restrictive than hypothesis (H3), is more likely to be acknowledged because experimental measurements of the coefficient on the boundary may be possible.
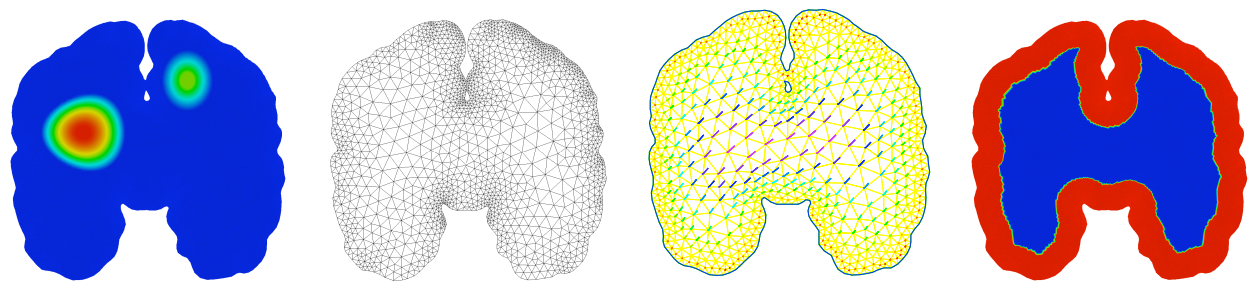

Figure 14. Unknown coefficient $\bar{p}$ (on the left), computational mesh and initial data $\bar{u}_{0}$ (in the center), observation zone $\omega$ (in red, on the right).

We can solve numerically the direct problem (1)-(3) by discretizing the equations

- in space using $\mathbb{P}^{1}$ Lagrange Finite Elements in the mesh shown on Figure 1,

- in time using a $\theta$-scheme with $\theta=0.5$ (implicit centered scheme) and $\delta t=1$,

- by using the trapezium formula for the integral term.

The time of observation is taken equal to $T=50$ whereas the observation zone $\omega$ is the one shown on Figure 1. A uniform relative error of $2 \%$ corresponding to the 
experimental error is added to the solution i.e. the observed displacement measured experimentally $u_{o b s}$ is obtained by the formula:

$$
u_{o b s}(x, t)=(1+r 0.02) \bar{u}(x, t), \quad \forall(x, t) \in \omega \times(0, T),
$$

where $r$ is a constant in $[-1,1]$, randomly chosen (according to a uniform distribution law) at each discretization point in the domain $\omega \times(0 ; T)$.

\subsection{Inverse problem}

We are looking for the minimizer of the non quadratic functional

$$
J(p)=\frac{1}{2} \int_{0}^{T} \int_{\omega}\left(\left|u-u_{o b s}\right|^{2}+\left|\nabla\left(u-u_{o b s}\right)\right|^{2}\right) d x d t,
$$

with $u=\mathcal{M}(p), \mathcal{M}$ being the nonlinear operator from $P$ to $U$ associated to system (1)-(3). In general, $u_{\text {obs }} \notin \mathcal{M}(P)$. Notice that adding a Tikhonov regularizing term to $J$ does not numerically help in this case since we will filter high frequencies when choosing the parameter space for $p$ (see section 5.4). We solve the minimization problem by a Broyden-Fletcher-Goldfarb-Shanno (BFGS) algorithm [31]. Thus, we calculate

$$
\begin{aligned}
\nabla J(p, \delta p) & =\lim _{\varepsilon \rightarrow 0} \frac{1}{\varepsilon}(J(p+\varepsilon \delta p)-J(p)) \\
& =\int_{0}^{T} \int_{\omega} M_{p} \delta p \cdot\left(\left(u-u_{o b s}\right)-\Delta\left(u-u_{o b s}\right)\right) d x d t,
\end{aligned}
$$

with $M_{p}$ the linearized operator of $\mathcal{M}$ around $p$, i.e. $M_{p} \delta p=\delta u$ satisfies

$$
\begin{cases}\mathcal{P} \delta u(x, t)=-\int_{0}^{t} \nabla \cdot\left(\delta p(x) h(t-s)\left(\nabla u(x, s)+\nabla u(x, s)^{T}\right)\right) d s, & \\ \delta u(x, 0)=0, & \forall x \in \Omega, \\ \partial_{t} \delta u(x, 0)=0, & \forall x \in \Omega, \\ \delta u(x, t)=0, & \forall(x, t) \in \partial \Omega \times(0, T) .\end{cases}
$$

We introduce the adjoint operator $\mathcal{P}^{*}$ of $(2)$

$$
\begin{aligned}
& \mathcal{P}^{*} v(x, t)=\partial_{t}^{2} v(x, t)-\nabla \cdot\left(\mu(x)\left(\nabla v(x, t)+\nabla v(x, t)^{T}\right)+\lambda(x)(\nabla \cdot v(x, t)) I\right) \\
& \quad+\int_{t}^{T} \nabla \cdot\left(p(x) h(s-t)\left(\nabla v(x, s)+\nabla v(x, s)^{T}\right)+\tilde{\lambda}(x, s-t)(\nabla \cdot v)(x, s) I\right) d s
\end{aligned},
$$

and we define $\delta u^{*}$, the solution of

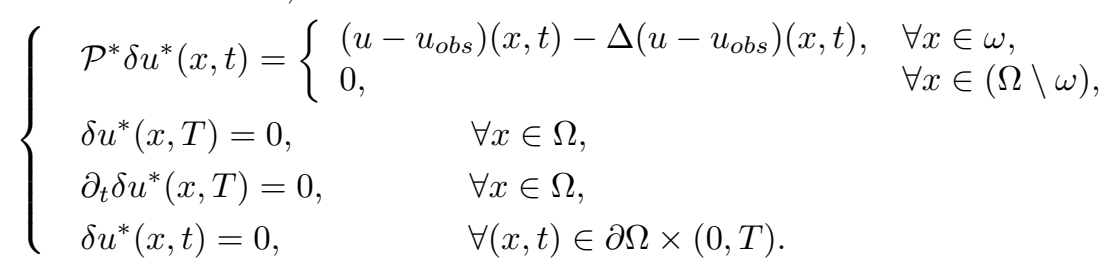

Therefore we can write:

$$
\begin{aligned}
\nabla J(p, \delta p) & =\int_{0}^{T} \int_{\Omega} \delta u(x, t) \cdot \mathcal{P}^{*} \delta u^{*}(x, t) d x d t=\int_{0}^{T} \int_{\Omega} \mathcal{P} \delta u(x, t) \cdot \delta u^{*}(x, t) d x d t \\
& =\int_{\Omega} \delta p(x)\left(\int_{0}^{T} \int_{0}^{t} h(t-s)\left(\nabla u(x, s)+\nabla u(x, s)^{T}\right): \nabla \delta u^{*}(x, t) d s d t\right) d x .
\end{aligned}
$$




\subsection{Regularization method}

We denote by $p^{*}$ the numerical parameter and by $\bar{p}$ the exact parameter, and we suppose $p^{*}$ close to $\bar{p}$. We can write:

$$
\left\|p^{*}-\bar{p}\right\|_{P}=\left\|M_{\bar{p}}^{-1}\left(M_{\bar{p}} p^{*}-\bar{u}\right)\right\|_{P} \leq\left\|M_{\bar{p}}^{-1}\right\|\left\|M_{\bar{p}} p^{*}-\bar{u}\right\|_{U} .
$$

If $M_{\bar{p}}$, linear from $P$ (of infinite dimension) to $U$, is compact for $\|\cdot\|_{P}$ and $\|\cdot\|_{U}$, then its inverse $M_{\bar{p}}^{-1}$ is not bounded. So, even if we find $p^{*}$ such that $M_{\bar{p}} p^{*}$ is close to $\bar{u}$, we cannot guarantee that $p^{*}$ will be close to $\bar{p}$. Thus, as regularization method [26], we introduce the operator

$$
R_{K}=\Pi_{K} M_{\bar{p}}^{-1}
$$

where $\Pi_{K}$ is the projection of $P$ to a finite dimensional space $P_{K}$. This operator $R_{K}$ is bounded in $U$ and verifies

$$
R_{K} \longrightarrow M_{\bar{p}}^{-1} \quad \text { if } K \rightarrow+\infty,
$$

and we now look for $p^{*} \in P_{K}$.

\subsection{Mesh and basis adaptation}

We choose to look for the unknown coefficient $p^{*}$ in the space $P_{K}$ of the $\mathrm{K}$ first eigenfunctions of the mesh, that is

$$
P_{K}=\left\{p \in P, p=\left.\bar{p}\right|_{\partial \Omega}+\sum_{i=1}^{K} p_{i} \varphi_{i}\right\}
$$

where $\left.\bar{p}\right|_{\partial \Omega}$ is a raising of the trace of the exact value of $\mathrm{p}$ (which is known as we assumed $\bar{p}$ known in $\omega$ ) and

$$
\begin{cases}-\Delta \varphi_{i}(x)=\sigma_{i} \varphi_{i}(x), & \forall x \in \Omega, \\ \varphi_{i}(x)=0, & \forall x \in \partial \Omega .\end{cases}
$$
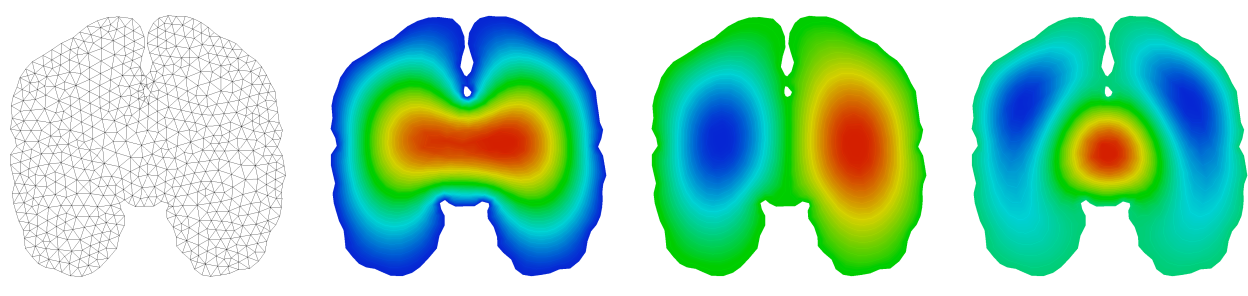

Figure 15. Initial mesh (left), initial basis functions $n^{o} 1, n^{o} 2$ and $n^{\circ} 5$ (right).

We propose an adaptive method to solve the problem accurately. After computing a first solution $p_{0}^{*}$ on the initial mesh, we use it to refine the mesh and to adapt the spectral basis. Indeed, we consider next as basis functions the solutions of the following problem:

$$
\begin{cases}-\nabla \cdot\left(a(x) \nabla \varphi_{i}(x)\right)=\sigma_{i} \varphi_{i}(x), & \forall x \in \Omega, \\ \varphi_{i}(x)=0, & \forall x \in \partial \Omega,\end{cases}
$$


with

$$
a(x)=\frac{1}{\nabla p_{0}^{*}(x)} .
$$

The initial and adapted meshes and basis functions are shown on Figure 15 and Figure 16
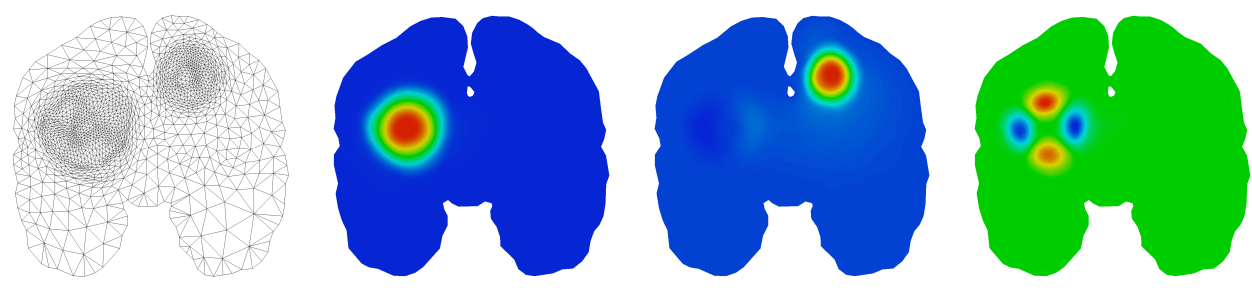

Figure 16. Adapted mesh (left), adapted basis functions $n^{o} 1, n^{o} 2$ and $n^{o} 5$ (right).

\subsection{The results}

We solve the inverse problem by the method introduced in 5.2-5.4. On Figure 17, we plot the relative error between $p^{*}$ and $\bar{p}$ in $L^{2}$-norm with respect to the number $K$ of eigenfunctions in the basis and for different steps of the iterative process.

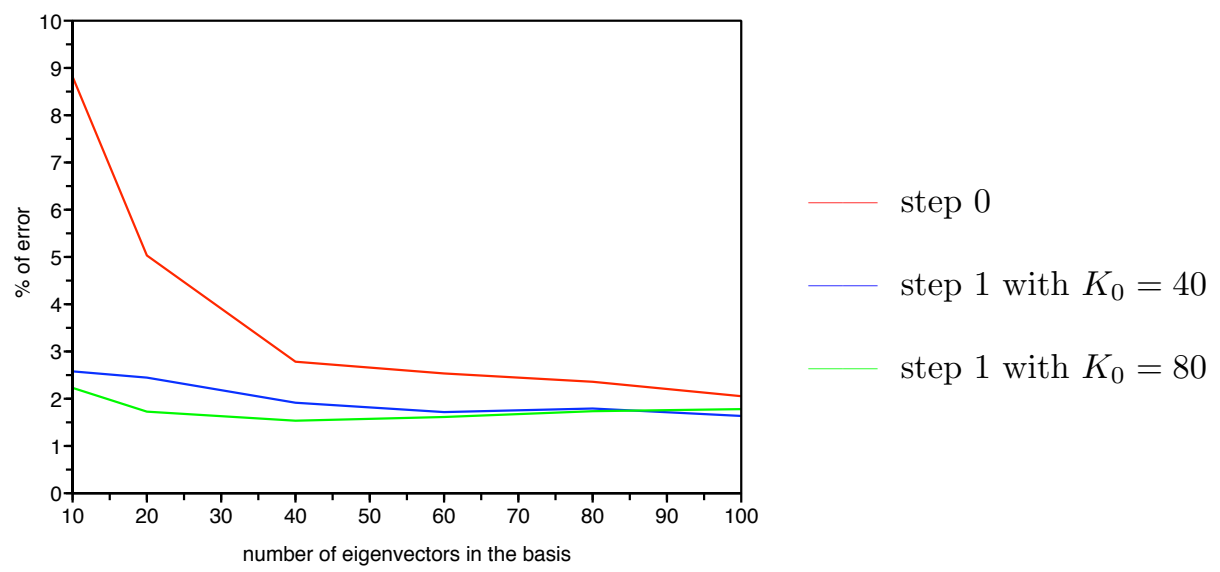

Figure 17. Relative error for the coefficient $p$ in $L^{2}$-norm with respect to $K$.

On Figure 18, we show the exact coefficient and the numerical result we obtain at different steps.

\subsection{Perspectives}

Notice that we only try to recover the coefficient $\bar{p}$, assuming that all the other coefficients are known. Indeed, the aim of this example is to illustrate the main result of the paper, which is the theoretical stability for recovering the spatial part of 


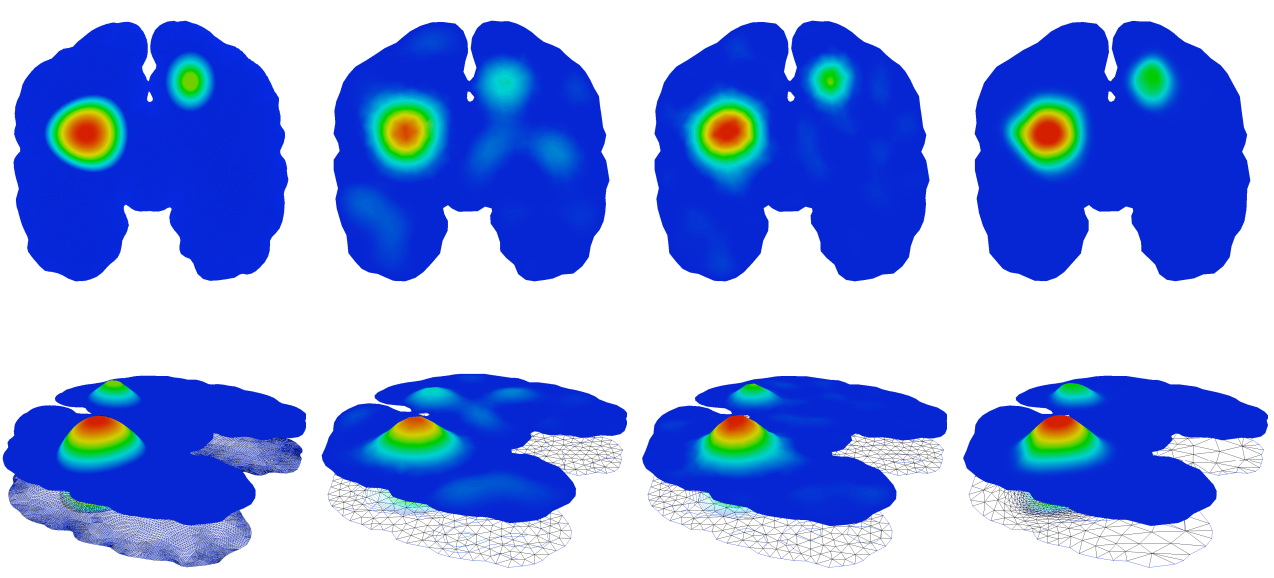

Figure 18. Exact coefficient $\bar{p}$ (on the left), recovered coefficient $p^{*}$ at step 0 with $K_{0}=50$ and $K_{0}=100$ (in the center), recovered coefficient $p^{*}$ at step 2 with $K_{0}=K_{1}=K_{2}=50$ (on the right).

only one viscoelastic coefficient from only one measurement. The other objective of this example is to show the interesting choice we can do for the spectral basis in an adaptive way. To recover simultaneously all the coefficients, there are various methods (see [32]). One method consists in adding to the minimal criteria more parameters, but this could be unstable if only a single observation is available, so we would need more independent observations. Another method consists in using an iterative procedure fixing all coefficients except one at each step. In both cases, we should start in a neighborhood of the real coefficients and play with noise levels. All this deep analysis of the numerical simulations is of course very relevant but it is out of the scope of the present publication. We are preparing a forthcoming publication that will include these numerical issues and more details about the choice of the spectral adaptive basis.

\section{Acknowledgments}

This work has been partially supported by the MathAmSud CIP-EDP project, by doctoral fellowships from CNRS and CONICYT for the first author and by the FONDECYT-CONICYT 1061263 grand for the second author.

\section{References}

[1] H. Bellout and J. Nečas. Existence of global weak solutions for a class of quasilinear hyperbolic integro-differential equations describing visco-elastic materials. Math. Ann., 299, 1994.

[2] M. Grasselli. Determining the relaxation tensor in linear viscoelasticity of integral type. Japan J. Ind. Appl. Math.,, 11, pp. 131-153, 1994.

[3] J. Janno and L. von Wolfersdorf. Inverse problems for identification of memory kernels in viscoelasticity. Mathematical Methods in the Applied Sciences, 20, pp. 291-314, 1997.

[4] L. von Wolfersdorf. On identification of memory kernels in linear viscoelasticity. Math. Nachrichten, 161, pp. 203-217, 1993.

[5] C. Cavaterra and M. Grasselli. On an inverse problem for a model of linear viscoelastic Kirchhoff plate. J. Integral Equations Appl., 9, pp. 179-218, 1997. 
[6] A. Lorenzi. A multidimensional identification problem related to a hyperbolic integrodifferential equation. Z. für Analysis und ihre Anwendungen, 18, pp. 407-435, 1999.

[7] A. Lorenzi and V.G. Romanov. Stability estimates for an inverse problem related to viscoelastic media. J. Inverse and Ill-Posed Problems, 18, pp. 407-435, 2006.

[8] A. L. Bukhgeim and M. V. Klibanov. Global uniqueness of class of multidimensional inverse problems. Soviet Math. Dokl., 24, pp. 244-247, 1981.

[9] T. Carleman. Sur un problème d'unicité pour les systèmes d'équations aux dérivées partielles à deux variables indépendantes. Ark. Mat. Astr. Fys., 2B, pp. 1-9, 1939.

[10] A. Lorenzi, F. Messina, and V. G. Romanov. Recovering a Lamé kernel in a viscoelastic system. Applicable Analysis, 86:11, pp. 1375-1395, 2007.

[11] M. M. Lavrent'ev. Some Ill-Posed Problems of Mathematics Physics. Berlin: Springer, 1967.

[12] M. de Buhan and A. Osses. Un résultat de stabilité pour la récupération d'un paramètre du système de la viscoélasticité 3D. C. R. Acad. Sci. Paris, Ser. I 347, pp. 1373-1378, 2009.

[13] M. Bellassoued. Global logarithmic stability in inverse hyperbolic problem by arbitrary boundary observation. Inverse Problems, 20, pp. 1033-1052, 2004.

[14] M. Bellassoued, O. Imanuvilov, and M. Yamamoto. Inverse problem of determining the density and the Lamé coefficients by boundary data. SIAM J. Math. Anal., 40, pp. 238-265, 2008.

[15] L. Hörmander. Linear Partial Differential Operators. Berlin, 1963.

[16] V. Isakov. Inverse Problems for Partial Differential Equations. Berlin: Springer, 1998.

[17] D. Tataru. Carleman estimates and unique continuation for solutions to boundary value problems. J. Math. Pures Appl., 75, pp. 367-408, 1996.

[18] O. Imanuvilov and M. Yamamoto. Global uniqueness and stability in determining coefficients of wave equations. Commun. Partial Diff. Eqns., 26, pp. 1409-1425, 2001.

[19] C. Cavaterra, A. Lorenzi, and M. Yamamoto. A stability result via Carleman estimates for an inverse source problem related to a hyperbolic integro- differential equation. Computational and Applied Mathematics, 25, pp. 229-250, 2006.

[20] M. V. Klibanov and A. Timonov. Carleman Estimates for Coefficient Inverse Problems and Numerical Applications. VSP, Utrecht, 2004.

[21] O. Imanuvilov and M. Yamamoto. Carleman estimates for the three-dimensional nonstationary Lamé system and applications to an inverse problem. Control Theory of Partial Differential Equations, Lect. Notes Pure Appl. Math., 242, pp. 337-374, 2005.

[22] O. Y. Imanuvilov. On Carleman estimate for hyperbolic equations. Asym. Anal., 32, pp. 185-220, 2002.

[23] M. Bellassoued. Unicité et contrôle pour le système de Lamé. ESAIM : Control, Optimisation and Calculus of Variations, 6, pp. 561-592, 2001.

[24] L. Robbiano. Fonction de coût et contrôle des solutions des équations hyperboliques. Asymptot. Anal., 10, 1995, pp. 95-115.

[25] J. Bros and D. Iagolnitzer. Tuboïdes et structure analytique des distributions. ii. Support essentiel et structure analytique des distributions. Séminaire Goulaouic-Lions-Schwartz 1974-1975: Equations aux dérivées partielles linéaires et non linéaires, Centre Math., Ecole Polytech., 18, 1975.

[26] A. Kirsch. An introduction to the mathematical theory of inverse problems. In Applied Mathematical Sciences, volume 120. Springer-Verlag, 1991.

[27] R. Dautray and J. L. Lions. Analyse mathématique et calcul numérique pour les sciences et les techniques, volume 3. Masson, 1987.

[28] O. Imanuvilov and J. P. Puel. Global Carleman estimates for weak solutions of elliptic nonhomogeneous Dirichlet problems. Int. Math. Res. Not., 16, pp. 883-913, 2003.

[29] G. Lebeau and L. Robbiano. Contrôle exact de l'équation de la chaleur. Comm. Partial Differential Equations, 20, pp. 335-356, 1995.

[30] J. C. Sarron, C. Blondeau, A. Guillaume, and D. Osmont. Identification of linear viscoelastic constitutive models. Journal of Biomechanics, 33, pp. 685-693, 2000.

[31] A. Modecai. Nonlinear Programming: Analysis and Methods. Dover Publishing, 2003.

[32] E. Pagnacco, A. Moreau, and D. Lemoss. Inverse strategies for the identification of elastic and viscoelastic material parameters using full-field measurements. Materials Science and Engineering, 452-453, pp. 737-745, 2007. 